\title{
DYNAMICAL MASSES OF YOUNG STARS. I. DISCORDANT MODEL AGES OF UPPER SCORPIUS
}

\author{
Aaron C. Rizzuto ${ }^{1}$, Michael J. Ireland ${ }^{2}$, Trent J. Dupur ${ }^{1}$, and Adam L. Kraus ${ }^{1}$ \\ ${ }^{1}$ Department of Astronomy, The University of Texas at Austin, Austin, TX 78712, USA \\ ${ }^{2}$ Research School of Astronomy \& Astrophysics, Australian National University, Canberra, ACT 2611, Australia \\ Received 2015 October 2; accepted 2015 December 16; published 2016 January 29
}

\begin{abstract}
We present the results of a long-term orbit monitoring program, using sparse aperture masking observations taken with NIRC2 on the Keck-II telescope, of seven G- to M-type members of the Upper Scorpius subgroup of the ScoCen OB association. We present astrometry and derived orbital elements of the binary systems we have monitored, and also determine the age, component masses, distance, and reddening for each system using the orbital solutions and multi-band photometry, including Hubble Space Telescope photometry, and a Bayesian fitting procedure. We find that the models can be forced into agreement with any individual system by assuming an age, but that age is not consistent across the mass range of our sample. The G-type binary systems in our sample have model ages of $\sim 11.5 \mathrm{Myr}$, which is consistent with the latest age estimates for Upper Scorpius, while the M-type binary systems have significantly younger model ages of $\sim 7 \mathrm{Myr}$. Based on our fits, this age discrepancy in the models corresponds to a luminosity underprediction of $0.8-0.15 \mathrm{dex}$, or equivalently an effective temperature overprediction of 100-300 K for M-type stars at a given pre-main-sequence age. We also find that the M-type binary system RXJ $1550.0-2312$ has an age ( $16 \mathrm{Myr})$ and distance $(\sim 85 \mathrm{pc})$ consistent with membership in the Upper Centaurus Lupus subgroup.
\end{abstract}

Key words: binaries: visual - stars: evolution - stars: fundamental parameters - stars: low-mass - stars: pre-main sequence

\section{INTRODUCTION}

The majority of the Galactic star formation is likely to occur in embedded clusters containing massive stars (Lada \& Lada 2003). Such embedded clusters dissipate and dissolve, leaving unbound $\mathrm{OB}$ associations. Stars within these $\mathrm{OB}$ associations, and in particular their subgroups, are thought to be a coeval population that share a common star formation history, chemical abundance, and velocity (de Zeeuw et al. 1999).

These young $\mathrm{OB}$ associations provide a glimpse into the state of a group of stars directly after formation. The nearest OB association to the Sun is the Scorpius-Centaurus-LupusCrux association (Sco-Cen) and is also our closest location of recent high-mass star formation $(\sim 150 \mathrm{pc})$. The association contains $\sim 150$ B-type stars that are spatially grouped into three subgroups: Upper Scorpius, Upper Centaurus Lupus (UCL), and Lower Centaurus Crux (LCC), and provides one of the richest nearby laboratories for the study of star and exoplanet formation (Rizzuto et al. 2011). The coeval Sco-Cen populations are often used as "age-calibrated" samples of objects in the study of a number of different science goals, such as circumstellar disk evolution (Carpenter et al. 2009; Chen et al. 2011; Rizzuto et al. 2012), exoplanet identification and evolution (Lafrenière et al. 2008, 2009; Ireland et al. 2011), and multiplicity studies (Kouwenhoven et al. 2007; Kraus et al. 2011; Rizzuto et al. 2013). Furthermore, mass estimation from models of very low mass companions to $\mathrm{K}$ - and M-type association members is highly dependent on the assumed age. Thus, it is critical that the age estimation for young associations is both highly accurate and unbiased.

The age of the Sco-Cen subgroups is contentious. The youngest and most compact subgroup, Upper Scorpius, was first age-dated using the main-sequence turnoff and was estimated to be $5-7$ Myr old (de Geus 1992). This age was supported by a spectroscopic survey for low-mass association members by Preibisch et al. (2002), which also determined a population age of $\sim 5 \mathrm{Myr}$ according to the latest models of the time, with a very narrow age spread. There is now some evidence for a spread in the HR diagram apparent age that correlates with the $\mathrm{Li}$ abundance (Rizzuto et al. 2015), and recent work utilizing new spectral typing and photometry of F-type members, and re-analysis of B/A-type members, has shown that Upper Scorpius may have a median age of $\sim 11 \mathrm{Myr}$, which is significantly different from any previous work (Pecaut et al. 2012).

There is still some uncertainty in the ages of the various B-type members of Upper Scorpius: while there are B-type Upper Scorpius stars that appear to have ages consistent with the $\sim 11$ Myr age estimation, there are others, such as $\tau$-Sco and $\omega$-Sco, that are clearly very young. In the case of $\tau$-Sco, this is verified independently as it has a well-determined temperature of $32,000 \pm 1000 \mathrm{~K}$, luminosity of $\log L / L_{\odot}=4.47 \pm 0.13$, and mass or $11 \pm 4 M_{\odot}$ measured from the $\mathrm{He}$ and $\mathrm{H}$ absorption lines (Simón-Díaz et al. 2006). Combined with the very slow rotation period of 43 days (Simón-Díaz et al. 2006; Strassmeier 2009), this means that $\tau$-Sco is certainly very young, with an age between 2 and $5 \mathrm{Myr}$ depending on the choice of models. For non-rotating stellar models, the most massive 15 Myr stars should be approximately $12 M_{\odot}$ (Ekström et al. 2012). The existence of stars like $\tau$-Sco and $\omega$-Sco cloud the clear picture of single-epoch star formation in the Upper Scorpius region and perhaps argue for some star formation between the age of $\rho$-Ophiuchus and the new canonical $\sim 11 \mathrm{Myr}$ age of Upper Scorpius.

The older subgroups of Sco-Cen also have somewhat unclear ages. The B-, A-, and F-type UCL and LCC members have main-sequence turnoff/turnon ages of $\sim 16-18 \mathrm{Myr}$ (Mamajek et al. 2002), while studies of the incomplete sample of lithium-rich G-, K-, and M-type members show a variety of 
Table 1

Keck-II/NIRC2 Orbit Monitoring Sample

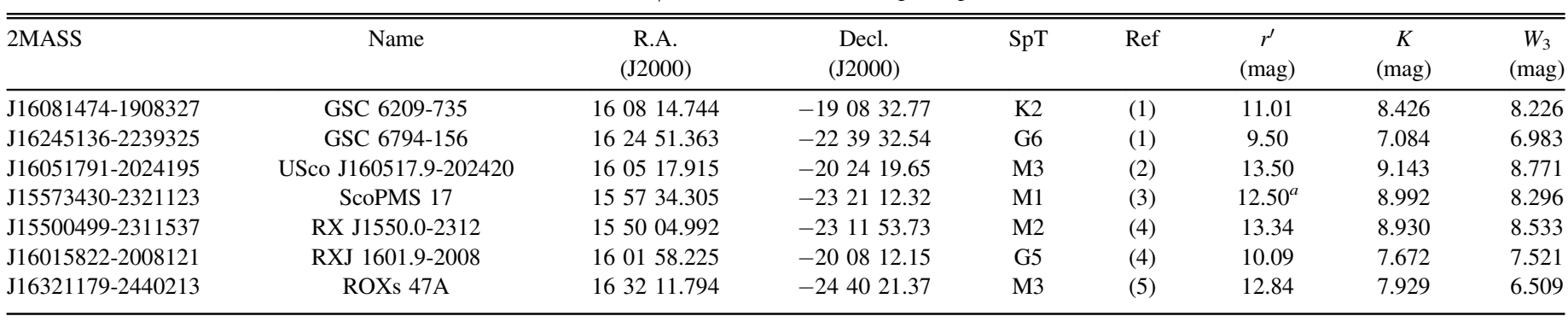

Note. Basic information for the stars in our orbit monitoring sample. The references for the spectral types are taken from: (1) Preibisch et al. (1998), (2) Preibisch et al. (2002), (3) Walter et al. (1994), (4) Köhler et al. (2000), (5) McClure et al. (2010). The $r^{\prime}$ magnitudes are taken from the APASS catalog, except for ScoPMS $17\left({ }^{a}\right.$ ), which is taken from USNO-A2 in the absence of an APASS measurement, and the $K$ magnitudes are taken from 2MASS. The final column lists the WISE band 3 $(12 \mu \mathrm{m}$ ) magnitudes (Wright et al. 2010). Note that with the exception of ROXs 47A, the sole Ophiuchus binary in our sample, these stars do not show evidence for the presence of a circumstellar disk (Luhman \& Mamajek 2012).

mass-dependent age estimates. The HR diagram age for the known K-type stars in UCL and LCC is $\sim 12 \mathrm{Myr}$, the few known M-type stars indicate a significantly younger age of $\sim 4 \mathrm{Myr}$, and the G-type members have an age of $17 \mathrm{Myr}$ that is consistent with the more massive stars (Preibisch \& Mamajek 2008; Song et al. 2012). There is also a positional trend in the age of the PMS stars of the older subgroups, with stars closer to the Galactic Plane appearing significantly younger than objects farther north. This is almost certainly the result of as yet undiscovered and unclarified substructure within the older subgroups, which have a very complex star formation history or unclear selection biases.

To date, the best large-scale age estimation for the Sco-Cen association and its subgroups has been purely photometric, meaning that colors and magnitudes are used to place association members on a theoretical HR diagram and then fit model isochrones. This method suffers from a number of issues beyond the inescapable model dependency. First, unknown binarity affects observed photometry: an equal mass binary will be 0.7 mag brighter than the either component, but will not change in color. In addition, fitting isochrones to photometry is highly dependent on the distance measure to the object being used. In particular, it has been shown that HIPPARCOS parallax measurements can potentially be incorrect for highmass binary systems (Tango et al. 2006). This is further complicated by the effect of the significant rotation of some B-type stars on HR diagram positioning.

Comparison of HR diagram positions of young stars to model isochrones also suffers from both the uncertainty intrinsic to the different evolutionary models and the uncertainty in the spectral type to effective temperature conversion for PMS stars. The various evolutionary models can predict masses differing by up to $50 \%$ for solar and sub-solar PMS stars (Hillenbrand \& White 2004; Stassun et al. 2014), and the uncertainty in the spectral type to effective temperature conversion for young stars can be as large as 100-200 K (Luhman et al. 2003).

A currently underutilized improvement for both age-dating Sco-Cen and evaluating the accuracy of PMS stellar evolution models at young ages is the inclusion of orbital mass information for well characterized Sco-Cen member binary system (e.g., Simon et al. 2013; Schaefer et al. 2014; Kraus et al. 2015. An accurate orbit can provide a direct measurement of the total system dynamical mass, which can then be used as an additional, orthogonal dimension in model fitting. Paired with one or more contrast ratios between the primary and the secondary companion at different wavelengths, this provides a vast improvement in estimating the age of individual stars, which will then provide indications of the age of the accompanying Upper Scorpius population.

In this paper, we present the orbits for seven low-mass Upper Scorpius stars monitored with Sparse Aperture Masking techniques with the NIRC2 Camera on the Keck-II telescope. Using these seven orbits we estimate stellar parameters for these binary systems, including age, component masses, and parallax, using a Bayesian model fitting algorithm. We then present some evaluation of the available PMS models for predicting mass and luminosity of young stars of different temperatures and provide an orbital estimate for the age of the Upper Scorpius subgroup of Sco-Cen.

\section{TARGET SAMPLE}

We present a sample consisting of six Upper Scorpius binary systems (two G-type, one K-type, and three M-type) and a single Ophiuchus region M-type binary system. These seven systems are the first completed orbits of a larger and ongoing orbit monitoring program of Sco-Cen, Taurus, and Ophiuchus binary members. Table 1 lists basic stellar properties for the seven binary systems. The six Upper Scorpius members in this study were selected from a multiplicity and planet-search survey of the Upper Scorpius subgroup of Sco-Cen (Kraus et al. 2008), the targets for which were compiled in Kraus \& Hillenbrand (2007) from the wider literature of membership surveys of the Upper Scorpius region (Walter et al. 1994; Preibisch et al. 1998, 2001, 2002; Ardila et al. 2000; Martín et al. 2004; Slesnick et al. 2006). The final object, ROXs 47A, a hierarchical triple system first identified by Barsony et al. (2003), is a member of the nearby $\rho$-Ophiuchus star-forming region that overlaps the Upper Scorpius region of sky. Following the identification of these systems as binary stars, all of these targets were flagged as ideal for orbit monitoring due to their small angular separations and projected periods that were on the order of a few years.

\section{KECK NIRC2 OBSERVATIONS AND ANALYSIS}

We have monitored the seven systems in our sample using NIRC2 aperture masking in natural guide star AO mode, on a yearly basis, over a timescale of approximately eight years. All NIRC2 AO observations were taken using the smallest 
Table 2

Keck-II/NIRC2 Astrometry

\begin{tabular}{|c|c|c|c|c|c|}
\hline Date & MJD & Filter & $\begin{array}{c}\rho \\
\text { (mas) }\end{array}$ & $\begin{array}{c}\theta \\
\left({ }^{\circ}\right)\end{array}$ & $\begin{array}{c}\Delta m \\
(\mathrm{mag})\end{array}$ \\
\hline \multicolumn{6}{|c|}{ GSC 6209-735 } \\
\hline 2014 Jul 30 & 56868 & $\mathrm{CH} 4 \mathrm{~S}$ & $17.2 \pm 1.7$ & $175.8 \pm 6.4$ & 3.05 (Fixed) \\
\hline 2013 Aug 7 & 56511 & $\mathrm{CH} 4 \mathrm{~S}$ & $16.4 \pm 2.5$ & $72.5 \pm 1.2$ & $2.90 \pm 0.50$ \\
\hline 2012 Apr 4 & 56021 & $\mathrm{CH} 4 \mathrm{~S}$ & $33.1 \pm 0.6$ & $31.8 \pm 0.7$ & $3.09 \pm 0.03$ \\
\hline 2009 Jun 1 & 54983 & $\mathrm{CH} 4 \mathrm{~S}$ & $26.1 \pm 1.6$ & $195.5 \pm 1.1$ & $3.50 \pm 0.10$ \\
\hline 2007 May 30 & 54250 & $\mathrm{CH} 4 \mathrm{~S}$ & $31.0 \pm 2.0$ & $42.5 \pm 3.6$ & $3.15 \pm 0.01$ \\
\hline \multicolumn{6}{|c|}{ GSC 6794-156 } \\
\hline 2015 Jun 23 & 57196 & $\mathrm{CH} 4 \mathrm{~S}$ & $63.0 \pm 0.1$ & $58.9 \pm 0.2$ & $0.51 \pm 0.01$ \\
\hline 2013 Aug 6 & 56510 & Kc & $70.5 \pm 0.1$ & $93.5 \pm 0.1$ & $0.46 \pm 0.01$ \\
\hline \multicolumn{6}{|c|}{ USco J160517.9-202420 } \\
\hline 2014 Jul 30 & 56868 & $\mathrm{CH} 4 \mathrm{~S}$ & $25.1 \pm 0.2$ & $303.8 \pm 0.6$ & $0.39 \pm 0.02$ \\
\hline 2012 Apr 4 & 56021 & $\mathrm{CH} 4 \mathrm{~S}$ & $31.73 \pm 0.04$ & $271.19 \pm 0.1$ & $0.44 \pm 0.01$ \\
\hline 2011 Jun 22 & 55734 & $\mathrm{CH} 4 \mathrm{~S}$ & $37.20 \pm 0.08$ & $278.6 \pm 0.1$ & $0.46 \pm 0.01$ \\
\hline 2010 Apr 5 & 55291 & $\mathrm{CH} 4 \mathrm{~S}$ & $38.28 \pm 0.07$ & $287.6 \pm 0.1$ & $0.39 \pm 0.01$ \\
\hline 2009 Jun 1 & 54983 & $\mathrm{CH} 4 \mathrm{~S}$ & $33.6 \pm 0.1$ & $294.3 \pm 0.2$ & $0.41 \pm 0.01$ \\
\hline 2008 Jun 17 & 54634 & $\mathrm{CH} 4 \mathrm{~S}$ & $21.4 \pm 0.1$ & $309.2 \pm 0.5$ & $0.52 \pm 0.02$ \\
\hline 2007 Jun 6 & 54257 & $\mathrm{~K}^{\prime}$ & $16.2 \pm 0.6$ & $251.1 \pm 1.1$ & $0.4 \pm 0.07$ \\
\hline \multicolumn{6}{|c|}{ ScoPMS 17} \\
\hline 2014 Aug 29 & 56867 & $\mathrm{Kc}$ & $34.4 \pm 0.1$ & $117.8 \pm 0.1$ & $0.71 \pm 0.01$ \\
\hline 2014 Jul 30 & 56868 & $\mathrm{CH} 4 \mathrm{~S}$ & $42.3 \pm 2.2$ & $136.7 \pm 3.0$ & $1.2 \pm 0.2$ \\
\hline 2012 Apr 4 & 56021 & $\mathrm{CH} 4 \mathrm{~S}$ & $66.96 \pm 0.20$ & $89.0 \pm 0.1$ & $0.86 \pm 0.01$ \\
\hline 2011 Jun 22 & 55734 & $\mathrm{CH} 4 \mathrm{~S}$ & $66.80 \pm 0.30$ & $77.5 \pm 0.3$ & $0.87 \pm 0.03$ \\
\hline 2010 Apr 5 & 55291 & $\mathrm{CH} 4 \mathrm{~S}$ & $58.66 \pm 0.15$ & $56.7 \pm 0.1$ & $0.89 \pm 0.01$ \\
\hline 2009 Jun 1 & 54983 & $\mathrm{CH} 4 \mathrm{~S}$ & $46.33 \pm 0.12$ & $36.0 \pm 0.1$ & $0.82 \pm 0.01$ \\
\hline 2008 Jun 17 & 54634 & $\mathrm{CH} 4 \mathrm{~S}$ & $26.88 \pm 0.17$ & $344.0 \pm 0.3$ & $0.81 \pm 0.01$ \\
\hline 2007 Jun 6 & 54257 & $\mathrm{~K}^{\prime}$ & $26.93 \pm 0.04$ & $222.1 \pm 0.1$ & $0.76 \pm 0.01$ \\
\hline 2007 Jun 5 & 54256 & $\mathrm{~K}^{\prime}$ & $26.95 \pm 0.05$ & $222.1 \pm 0.1$ & $0.76 \pm 0.01$ \\
\hline \multicolumn{6}{|c|}{ RXJ 1601.9-2008 } \\
\hline 2015 Jun 23 & 57196 & $\mathrm{CH} 4 \mathrm{~S}$ & $38.0 \pm 0.5$ & $215.1 \pm 0.7$ & $2.0 \pm 0.04$ \\
\hline 2014 Jul 29 & 56867 & $\mathrm{~K}^{\prime}$ & $32.8 \pm 0.4$ & $206.1 \pm 0.3$ & $1.84 \pm 0.02$ \\
\hline 2012 Apr 4 & 56021 & $\mathrm{CH} 4 \mathrm{~S}$ & $17.5 \pm 1.7$ & $100.5 \pm 1.2$ & $2.3 \pm 0.3$ \\
\hline 2011 Jun 22 & 55734 & $\mathrm{CH} 4 \mathrm{~S}$ & $25.5 \pm 0.3$ & $67.6 \pm 0.3$ & $2.04 \pm 0.02$ \\
\hline 2010 Apr 5 & 55291 & $\mathrm{CH} 4 \mathrm{~S}$ & $28.5 \pm 0.4$ & $43.8 \pm 0.3$ & $2.08 \pm 0.02$ \\
\hline 2008 Jun 17 & 54634 & $\mathrm{CH} 4 \mathrm{~S}$ & $24.4 \pm 0.7$ & $231.5 \pm 0.8$ & $2.08 \pm 0.05$ \\
\hline 2007 May 31 & 54251 & $\mathrm{CH} 4 \mathrm{~S}$ & $39.3 \pm 1.6$ & $217.7 \pm 0.6$ & $2.1 \pm 0.1$ \\
\hline \multicolumn{6}{|c|}{ ROXs 47A } \\
\hline 2014 Jul 29 & 56867 & $\mathrm{~K}^{\prime}$ & $52.3 \pm 0.2$ & $73.1 \pm 0.2$ & $0.09 \pm 0.01$ \\
\hline 2013 Aug 7 & 56511 & $\mathrm{CH} 4 \mathrm{~S}$ & $42.39 \pm 0.04$ & $62.2 \pm 0.1$ & $0.37 \pm 0.01$ \\
\hline 2011 Jun 22 & 55734 & $\mathrm{~J}$ & $21.2 \pm 0.3$ & $141.1 \pm 1.6$ & $0.21 \pm 0.02$ \\
\hline 2010 Apr 5 & 55291 & $\mathrm{CH} 4 \mathrm{~S}$ & $43.4 \pm 0.2$ & $108.5 \pm 0.2$ & $0.22 \pm 0.01$ \\
\hline 2009 Jun 1 & 54983 & $\mathrm{CH} 4 \mathrm{~S}$ & $51.8 \pm 0.2$ & $98.7 \pm 0.2$ & $0.17 \pm 0.01$ \\
\hline
\end{tabular}


Table 2

(Continued)

\begin{tabular}{lccccc}
\hline \hline Date & MJD & Filter & $\begin{array}{c}\rho \\
(\mathrm{mas})\end{array}$ & $\begin{array}{c}\theta \\
\left({ }^{\circ}\right)\end{array}$ \\
\hline 2002 May $24^{a}$ & 52418 & $\mathrm{~K}^{\prime}$ & $40 \pm 30$ & $107 \pm 20$ \\
\hline
\end{tabular}

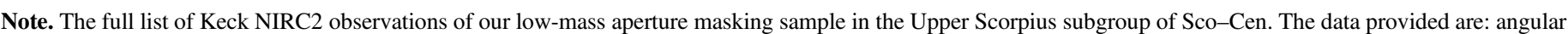

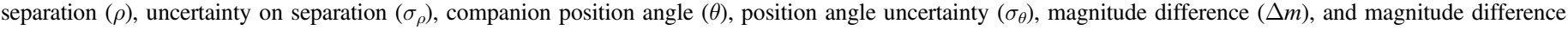

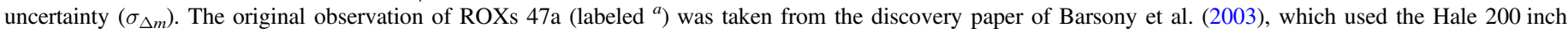
telescope; this measurement was not included in the orbital fit.

available pixel scale of $9.95 \mathrm{mas}_{\mathrm{pixel}}{ }^{-1} \quad$ (Yelda et al. 2010) and either a two or four location dither pattern. The majority of the observations were taken with the nine-hole aperture mask and the narrowband $\mathrm{CH}_{4} \mathrm{~S}$ filter, with some observations in $J, K^{\prime}$, and $L^{\prime}$.

Aperture masking data reduction utilizes the complex triple product or closure phase, in addition to squared interferometric visibilities, in order to remove non-common path errors and variable optical aberrations. Binary system profiles can then be fit to the visibilities and closure phases to produce separations and position angles. A full explanation of the reduction and closure-phase fitting procedure is given in the appendix of Kraus et al. (2008). In Table 2, we list the individual aperture masking observation details, including observation filter, fitted separations, and position angle.

\section{ORBIT FITTING}

The astrometric measurements taken in our orbit monitoring program were used to determine orbital solutions using a $\chi^{2}$ minimization on a grid of orbital parameters. We first generated a random sample of $10^{4}$ sets of semimajor-axis, eccentricity, and system mass values. The range over which the semimajor-axis samples were taken spanned 0.5-1.5 times the maximum observed separation of the each binary system, while the eccentricity was sampled in the range $0<e<1$. We chose the system mass sample range based on the spectral types in Table 1, with a conservatively large range of possible masses spanning $0.1-3 M_{\odot}$. From each random system mass and semimajor-axis pair, we calculate the corresponding period, using the characteristic distance of Upper Scorpius according to Kepler's law $M_{\text {tot }}=a^{3} P^{-2}\left(d_{\mathrm{US}}^{3}\right)$. Using this random sample, we then fit the remaining four orbital parameters. We then further restrict the possible range of the randomly selected parameters and draw a new random sample centered on the current best-fit values, and the process is repeated. The best-fit parameters from this second sampling are then taken as the starting point for a Monte Carlo Markov Chain process to more precisely determine the best-fit orbital parameters.

Table 3 lists the orbital solutions for the stars in our program, while the corresponding orbital plots can be found in Figures 1 and 2. Typically, the semimajor axis (in angular units) and the period, the two important parameters for estimation of the system dynamical mass, are determined to better than $\sim 2 \%-$ $3 \%$. In the last two columns of Table 3, we list the system dynamical masses at a fixed parallax of 7.5 mas, chosen to represent the mean distance to the Upper Scorpius subgroup. These system masses can then be appropriately scaled to an alternate parallax $\pi_{n}$ through multiplication by $\left(7.5 / \pi_{n}\right)^{3}$, with appropriately adjusted uncertainties.
One of the tightest orbits that we have determined has also been identified as a single-lined spectroscopic binary by Guenther et al. (2007). The RV orbit has a period of $2045 \pm 16$ days and eccentricity of $0.2 \pm 0.03$ that are very similar to our orbital period measurement of $1998.4 \pm 18.3$ days and eccentricity of $0.219 \pm 0.014$. Because the distance to these stars is unknown, the mass ratio of the primary and secondary components cannot be directly disentangled from the combination orbital solutions; however, the mass function from the RV orbit, $f_{m}=0.0049 \pm 0.0005$, can be included as additional information with which to fit models to the orbital and photometric data.

\section{Hubble Space Telescope (HST) OBSERVATIONS}

In addition to AO imaging, we have obtained single-epoch observations of these binary systems with the HST Wide Field Camera 3 (WFC3), in a variety of visible filters spanning wavelengths of $200-1000 \mathrm{~nm}$. We took either three or four exposures in each filter, to which was applied the standard $H S T$ reduction, calibration, and cosmic-ray rejection procedure (Rajan 2010). We calculated binary system unresolved magnitudes for each WFC3 filter using aperture photometry on the HST drizzle images with a 0. " 4 radius star aperture and a sky annulus of 4 " -6 ". We used a 0 ". 4 aperture to facilitate calibration with the readily available WFC3 filter information and to ensure that the wide companion in the ROXs 47A triple system was excluded from the both the star aperture and the sky subtraction region. For GSC 6794-156, the images had the center of the star PSF flagged (most likely incorrectly) as cosmic rays in all three uncombined FLT images, and so for this star the combined drizzle images were expected to give incorrect photometry. For this binary system we applied the aperture photometry procedure to the uncombined FLT images.

Given the orbital solutions we have determined for these systems, we can accurately predict the separation and position angle of each binary system at the epoch of observation with the HST to within a few milliarcseconds. Combined with the stability of the HST point-spread function (PSF), we can derive differential photometry from our observations even though the binary separations are typically $<60$ mas. Our PSF fitting is modeled after our previous work (Garcia et al. 2015), with the addition of a further complication due to the short exposure times used in many of the filters in which our sample was observed. For the majority of the observations, we used three exposures that were often shorter than $1 \mathrm{~s}$, an exposure regime in which vibrations induced by the WFC3 shutter causes observable blur in the images. It has been shown in on-sky and laboratory tests that following the rotation of the WFC3 shutter at the beginning of an exposure, there is a period of a few seconds in which the PSF shape is degraded due to vibrations in the camera (Hartig 2008). In order to account for the 
Table 3

Astrometric Orbital Elements

\begin{tabular}{|c|c|c|c|c|c|c|c|c|}
\hline Star & $\begin{array}{c}T_{0} \\
\text { (JD) }\end{array}$ & $\begin{array}{c}P \\
\text { (days) }\end{array}$ & $\begin{array}{c}a \\
\text { (mas) }\end{array}$ & $\epsilon$ & $\begin{array}{l}\Omega \\
\left({ }^{\circ}\right)\end{array}$ & $\begin{array}{l}\omega \\
\left(^{\circ}\right)\end{array}$ & $\begin{array}{c}i \\
\left({ }^{\circ}\right)\end{array}$ & $\begin{array}{l}M_{\text {tot }} \\
\left(M_{\odot}\right)\end{array}$ \\
\hline GSC 6209-735 & $2455182.8 \pm 3.5$ & $2015.5 \pm 12.6$ & $28.22 \pm 0.47$ & $0.210 \pm 0.012$ & $207.7 \pm 1.1$ & $27.3 \pm 1.8$ & $64.7 \pm 1.1$ & $2.25 \pm 0.11( \pm 0.41)$ \\
\hline GSC 6794-156 & $2453837.3 \pm 4.9$ & $4851.4 \pm 29.8$ & $58.19 \pm 0.31$ & $0.262 \pm 0.004$ & $328.6 \pm 1.4$ & $45.2 \pm 1.5$ & $161.4 \pm 1.2$ & $3.40 \pm 0.02( \pm 0.62)$ \\
\hline J160517.9-202420 & $2456553.5 \pm 0.4$ & $2146.2 \pm 3.4$ & $21.88 \pm 0.38$ & $0.907 \pm 0.004$ & $160.4 \pm 5.7$ & $59.3 \pm 5.8$ & $154.8 \pm 2.0$ & $0.92 \pm 0.05( \pm 0.17)$ \\
\hline ScoPMS 17 & $2455945.3 \pm 1.0$ & $5471.5 \pm 47.1$ & $47.58 \pm 0.37$ & $0.880 \pm 0.002$ & $199.6 \pm 0.3$ & $286.1 \pm 0.3$ & $124.0 \pm 0.3$ & $1.46 \pm 0.02( \pm 0.26)$ \\
\hline RX J1550.0-2312 & $2454412.5 \pm 0.5$ & $3195.7 \pm 12.9$ & $46.58 \pm 0.06$ & $0.488 \pm 0.001$ & $241.6 \pm 0.8$ & $30.5 \pm 1.0$ & $29.1 \pm 0.6$ & $4.03 \pm 0.04( \pm 0.73)$ \\
\hline RX J1601.9-2008 & $2454974.5 \pm 3.2$ & $2983.3 \pm 20.5$ & $36.18 \pm 0.27$ & $0.356 \pm 0.012$ & $223.6 \pm 0.5$ & $106.9 \pm 0.7$ & $72.7 \pm 0.4$ & $2.16 \pm 0.05( \pm 0.38)$ \\
\hline ROXs 47A & $2455929.8 \pm 1.9$ & $3007.1 \pm 42.9$ & $32.69 \pm 0.35$ & $0.818 \pm 0.009$ & $24.1 \pm 11.0$ & $243.0 \pm 10.9$ & $16.3 \pm 2.0$ & $1.57 \pm 0.03( \pm 0.29)$ \\
\hline
\end{tabular}

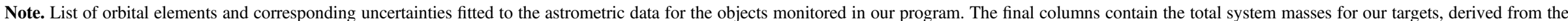

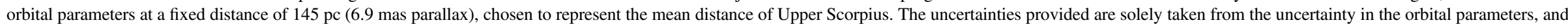

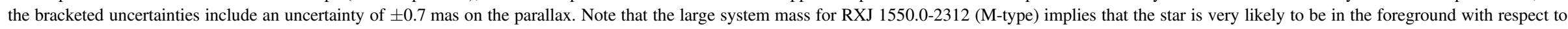
Upper Scorpius, with a parallax greater than $\sim 10$ mas. 


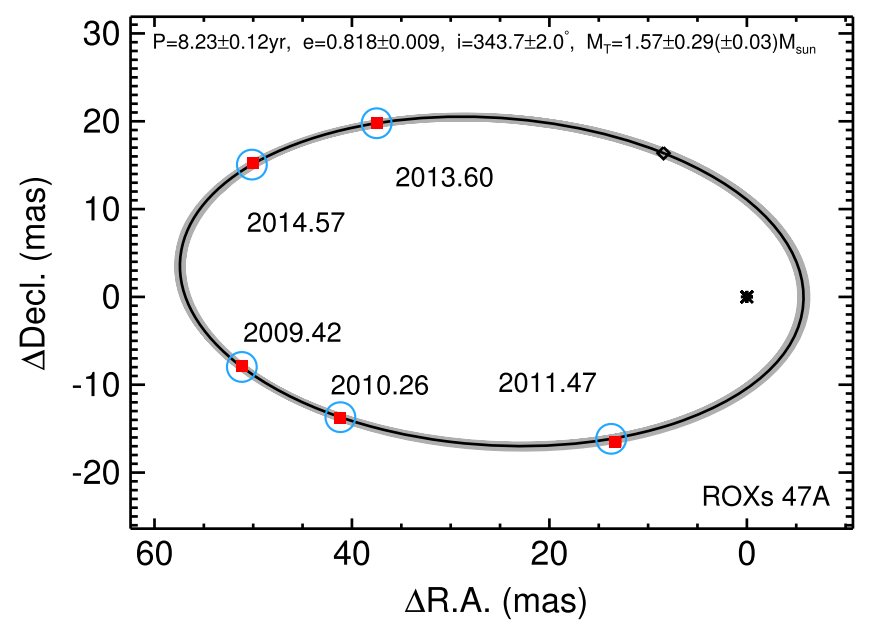

Figure 1. Orbital solution for Ophiuchus binary ROXs 47Aab from NIRC2 AO aperture masking. The data are shown as red squares, with corresponding model fits shown as blue circles. We show the best-fit orbit in black, with a $1 \sigma$ region shaded in gray. The black diamond shows the orbital position of the secondary at the time of the corresponding HST observations (see Table 5). We also display the orbital period, semimajor axis, eccentricity, and system mass at $145 \mathrm{pc}$. The two uncertainties listed for the mass are derived by either including or excluding the $\pm 15 \mathrm{pc}$ distance uncertainty associated with Upper Scorpius membership. The bracketed uncertainty, which only includes the uncertainty on the orbital parameters, is representative of the system mass precision attainable with future GAIA parallaxes.

presence of the vibrational blurring on the output contrast ratios, we both employed a large library of unblurred PSF standards and characterized the effect of the vibration on the photometry of a binary system. First, we used a large library of archival data with long exposures to identify multiple unblurred PSF reference sources in each filter of interest for both the C512C and M512C subarrays of the UVIS2 detector. Using the Tiny Tim software (Krist et al. 2011), we created PSF models for the WFC3 filters; these were then fit to the PSF standards to determine a modified PSF that most closely fits the large sample of reference data. This process involved vetting the PSF references for obvious $>1$ pixel binaries and sources where cosmic rays landed within a few pixels of the target center. All other contaminants outside of a four pixel radius from the PSF center were handled automatically via sigma clipping with respect to the residual distribution of the entire PSF reference library.

We created a set of synthetic, blurred binary systems at different integer-pixel separations and contrast ratios, using multiple images of ROXs $47 \mathrm{~B}$, the wide tertiary companion to ROXs 47A that was in the frame of the ROXs 47A images. We then convolved the reference model PSF in each filter, created as described above, with a Gaussian kernel and attempted to recover the original input contrast ratios. To do this, we fixed the separation and P.A. of the synthetic binaries and allowed the position of the primary star, flux normalization, flux ratio, and blur FWHM to vary. With this method, we were able to create a grid of photometry corrections at different separations for both the mild and severely blurred images by fitting polynomials as a function of output contrast ratios. These corrections were then applied to the PSF fits for the binary systems in our sample by interpolating on a 3D grid of position and blur Figure 3 displays an example binary fit residuals for the blurred and unblurred models, and Figure 4 displays an example contrast ratio correction for one of the synthetic binary systems. The uncertainties for the magnitude differences were taken as the rms between the images in each filter and the uncertainty on the correction combined in quadrature. The aperture and differential photometry for our sample is presented in Tables 4 and 5, respectively.

\section{BINARY SED PROPERTIES}

We have used the unresolved WFC3 and 2MASS photometry, combined with the resolved magnitude differences from the NIRC2 aperture masking observations to determine temperatures and luminosities for the components of the binary systems in our monitoring sample. We employed a grid of paired BT-Settl (Allard \& Hauschildt 1995; Baraffe et al. 2015) synthetic spectra, convolved with a filter profile for each of the WFC3 and 2MASS filters, and compared these to the observed values using a $\chi^{2}$ methodology. There is some degeneracy between extinction and temperature, so we allowed $A_{V}$ to vary from 0.6 to $1.2(0.24<E(B-V)<0.36)$, which is a typical range of values for Upper Scorpius members (Preibisch et al. 2002; Rizzuto et al. 2015). For ROXs 47A, the single Ophiuchus member in our sample, we doubled the prescribed reddening in anticipation of stronger extinction toward the starforming region. To determine the component temperatures, we take both BT-Settl atmospheres and combine them at a given ratio, and then scale the resulting combined spectra to best match the observed photometry, repeating the process for a generous range of effective temperatures. Figure 5 displays the SED fit for GSC 6794-156 as an example. The primary and secondary bolometric fluxes are then calculated for the individual best-fit temperatures, excluding the effects of extinction. With the exception of GSC 6209-735 and RX J1601.9-2008, the two highest contrast binary systems in our sample, we were able to determine individual component temperatures using this method. The uncertainty in the derived temperatures and bolometric fluxes are both strongly dominated by the unconstrained extinction.

Given these estimates for the component fluxes and temperatures, we can now place these PMS stars on the HR diagram and derive age and mass estimates from the data. Upper Scorpius inhabits a significant volume of space, with a depth of \pm 15 pc (de Zeeuw et al. 1999; Rizzuto et al. 2011). This comprises a significant uncertainty in determining HR diagram luminosities for the binary systems in our sample. However, the system dynamical mass observable, derived from the binary system orbital parameters, provides an indirect constraint on the distance of the binary system. As mentioned above, the dynamical mass observable for an astrometric orbit $M_{\mathrm{tot}} d^{-3}=a^{3} P^{-2}$ is strongly dependent on the binary system distance and can be used to better constrain the HR diagram position of the binary system components. For each system, we then fit for distance and component masses as a function of the estimated temperatures and luminosities and the measured dynamical mass observable. For the two systems with unconstrained secondary luminosity and temperature, we conservatively fix the secondary mass in the fit to be $0.5 \pm 0.3 M_{\odot}$ and fit for the primary mass and system distance. Figure 6 displays the HR diagrams for both the G-/K- and M-type binary systems in our sample, and the resulting properties of the components are listed in Table 6.

The two G-type members, GSC 6794-156 and GSC 6209735, and RXJ 1601.9-2008 all have HR diagram ages of $\sim 10 \mathrm{Myr}$, which agrees directly with the most recent age measurement of Pecaut et al. (2012). The M-type binaries 

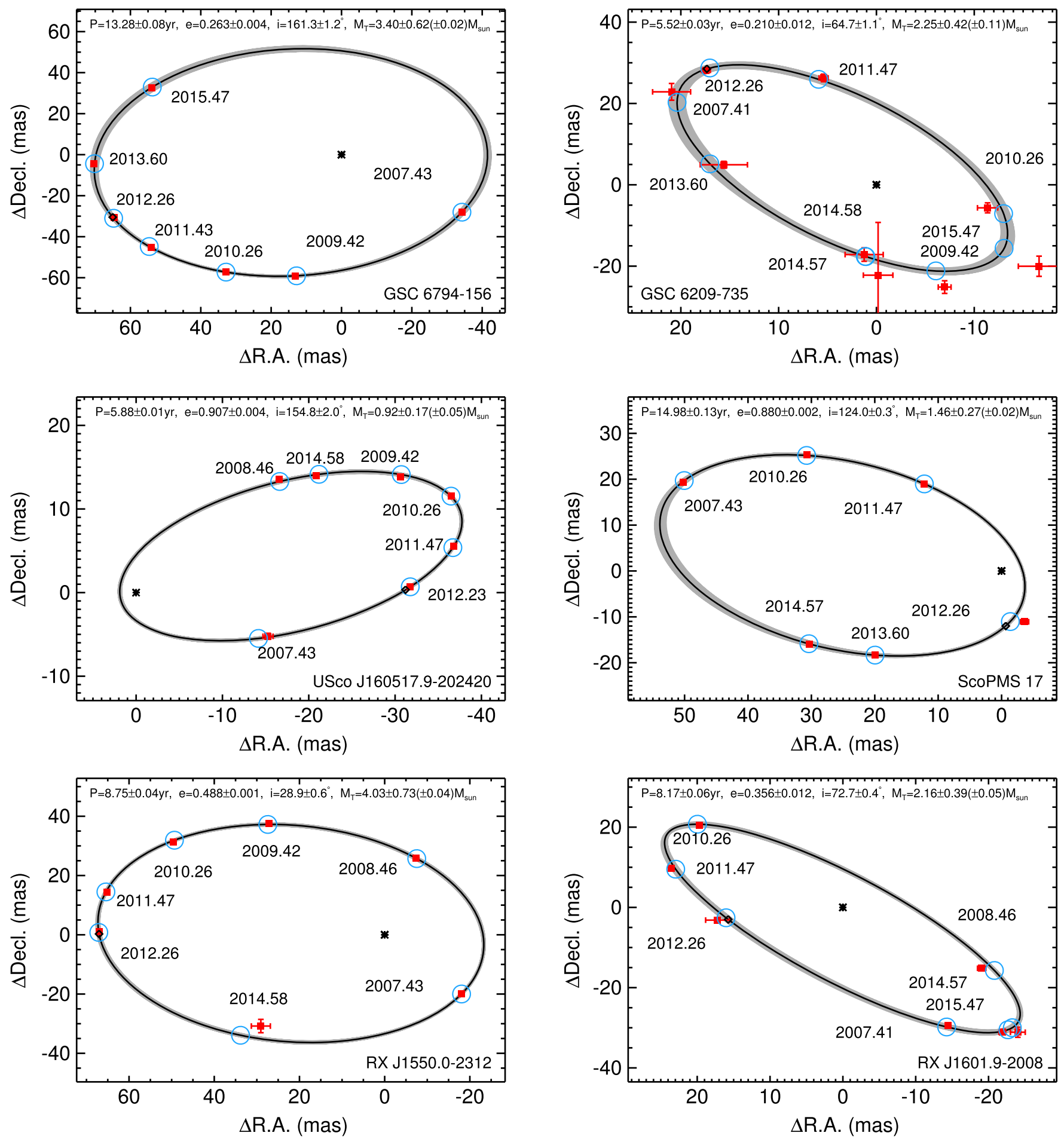

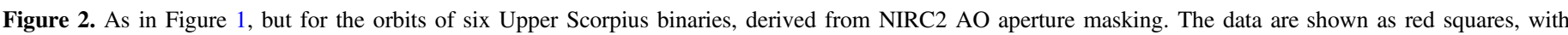

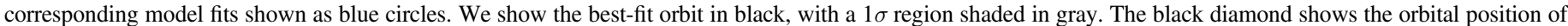

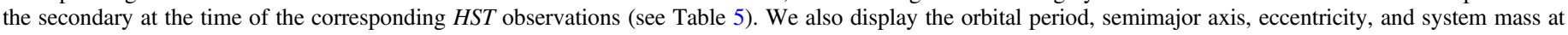

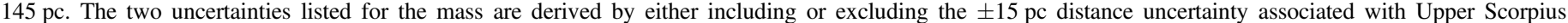

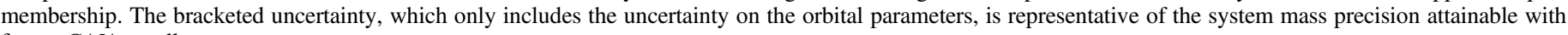
future GAIA parallaxes.

ScoPMS 17 and USco J160517.9-202420 both appear slightly younger than the G-type binaries, with HR diagram ages of $\sim 7$ Myr. We note that RXJ 1550.0-2312 appears significantly older than the other M-type binary system in our sample, with an age more consistent with Upper Centaurus Lupus membership.

\section{BAYESIAN AGE ESTIMATION}

While the SED fitting and HR diagram position estimates for the binary component properties produce useful results in characterizing both the individual systems and the overall population in Upper Scorpius, the uncertainties introduced due 


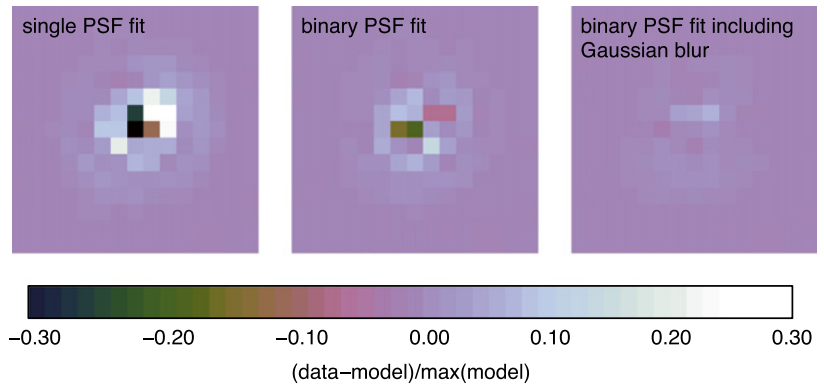

Figure 3. Example PSF fit residuals for the binary system RXJ 1550.0-3212, in the filter F775W and the M512C subarray. From left to right, panels show the residuals when fitting a single PSF, binary PSF, and binary PSF with Gaussian blur to account for the shutter vibration. At the epoch of this $H S T$ observations, the binary components were separated by 65.8 mas at a position angle of $90{ }^{\circ} 1$ $(d x, d y=1.46,0.8$ pixels $)$

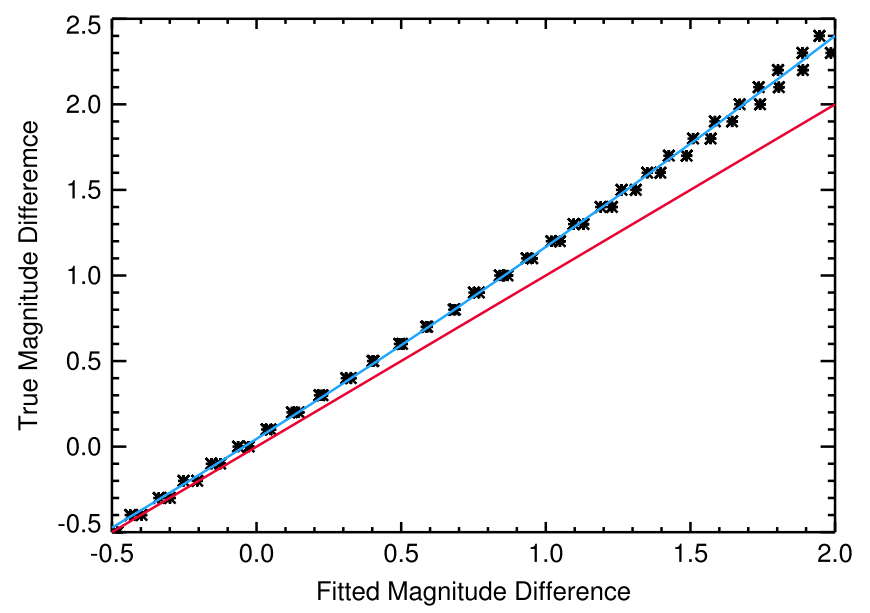

Figure 4. Contrast ratio correction for a synthetic binary separation ( $x$ $y)=-(2,2)$ pixels on the WFC3 detector. The black stars are the fitted contrast ratios, the blue line is the polynomial fit used to correct the actual binary system data, and the red line is the case for a zero correction.

to the unconstrained extinction warrant a more sophisticated approach that uses all available information to simultaneously determine the relevant parameters. The goal is to produce a method for determining the age, masses, distance, and extinction for the stars in a binary system of known orbit, drawing data from the orbital parameters and measured magnitudes in a number of filters, as well as a contrast ratio in one or more filters. We phrase the problem in terms of Bayes's Theorem:

$$
P(\Phi \mid D) \propto P(\Phi) P(D \mid \Phi),
$$

where $\Phi$ represents a model and $D$ represents the data. The model $\Phi$ consists of an age, model parallax $\left(\pi_{m}\right)$, primary and secondary masses $\left(M_{p}\right.$ and $\left.M_{s}\right)$, a reddening parameter $(E(B-V))$, and a set of isochrones, which map mass, reddening, and age to magnitudes in different filters which can be compared to the data.

The data, $D$, consist of an association parallax in the absence of a directly measured distance $(7.5 \pm 1.7$ mas, taken from Rizzuto et al. 2011); the total mass observable $\left(M_{T} \pi^{3}=a^{3} / P^{2}\right)$ calculated from the orbital period and semimajor axis; the magnitude difference in one or more filters $\left(\Delta m_{i}\right)$, taken from our AO aperture masking observations and HST photometry; and a set of unresolved magnitudes in available catalog filters $\left\{m_{\star, i}\right\}$, including APASS BVgri filters, 2MASS $J, H$, and
$K$ and HST WFC3 filters. The treatment of the total mass observable $\left(M_{T} \pi^{3}=a^{3} / P^{2}\right)$ and its corresponding uncertainty requires some care because the fitter orbital period $(P)$ and semimajor axis $(a)$ are often highly correlated. To properly compute the uncertainty in the total mass observable, we take the full covariance matrix of the orbital parameters and use the coordinate transform to recast it in the total mass observable coordinates.

In the fortuitous case where both a visual orbit and a radial velocity orbit (either single or double lined) are available simultaneously for a single binary system, we can incorporate the additional radial velocity information, such as mass ratio for the double-lined spectroscopic orbit or the primary mass function $(f(M))$. Incorporation of the mass ratio into the following framework is trivial; however, the inclusion of the mass function, as is the case for GSC 6209-735, requires some description. We combine the mass function $(f(M))$ with the inclination taken from the visual orbit in order to define a mass function observable $M_{f(M)}$ :

$$
M_{f(M)}=\frac{M_{s}^{3}}{\left(M_{p}+M_{s}\right)^{2}}=\frac{f(M)}{\sin i^{3}} .
$$

To compute the Bayesian probability, we first expressed the models in terms of the more directly comparable parameters such as magnitude difference and unresolved magnitude using marginalization:

$$
P(D \mid \Phi)=P(D \mid \phi) P(\phi \mid \Phi),
$$

where

$$
\Phi=\left\{\text { Age }, \pi_{m}, M_{p}, M_{s}, m_{p, i}, \mathrm{~m}_{s, i}\right\},
$$

$\left.\phi=\left\{\pi_{m}, m_{s+p, i}, \Delta m_{i},\left(M_{p}+M_{s}\right) \pi_{m}^{3}, M_{s}^{3}\left(M_{p}+M_{s}\right)^{-2}\right)\right\}$, and $\Delta m_{i}$ is the magnitude difference between secondary and primary in filter $i$ for the given primary and secondary masses. $m_{s+p, i}$ is the unresolved magnitude in filter $i$ of the primary and secondary for the given masses and at the given parallax, reddened according to the Savage \& Mathis (1979) extinction law and the model reddening parameter. We have included in this description the mass function observable because it is used for the binary system GSC 6209-735 for which we have a mass function measurement; however, an additional term for the mass ratio can be easily added to the formality above.

Note that the above transformation is simple because the new parameters are directly given by the original model, and so $P(\phi \mid \Phi)=1$, leaving the following:

$$
P(\phi \mid D)=\frac{P(D \mid \phi) P(\Phi)}{P(D)} .
$$

For the prior probability distribution $P(\Phi)$, we have chosen a uniform distribution, meaning that all values of the model parameters are initially treated as being equally likely. For each set of model parameters, we then calculate $P(D \mid \phi)$, which takes the following form when separated into individual variables:

$$
\begin{aligned}
& P(D \mid \phi)=P\left(\pi_{\star} \mid \pi_{m}\right) P\left(M_{t} \pi^{3} \mid\left(M_{p+s}\right) \pi_{m}^{3}\right) \\
& P\left(M_{f(M)} \mid M_{s}^{3} M_{p+s}^{-2}\right) \\
& \prod_{i} P\left(m_{\star, i} \mid m_{s+p, i}\right) \prod_{j} P\left(\Delta m_{\star, j} \mid \Delta m_{j}\right),
\end{aligned}
$$

where $i$ and $j$ indicate multiplication over all available photometric filters. The probabilities in the above equations are modeled as normal distributions, with standard deviation 
Table 4

HST/WFC3 Unresolved Photometry

\begin{tabular}{|c|c|c|c|c|c|c|c|}
\hline Filter & RXJ $1550 \ldots$ & RXJ $1601 \ldots$ & USco J1605... & GSC 6209-735 & GSC 6794-156 & ROXs 47A & ScoPMS017 \\
\hline F225W & $\ldots$ & $14.96 \pm 0.04$ & $\ldots$ & $\ldots$ & $14.34 \pm 0.03$ & $\ldots$ & . \\
\hline F275W & $18.43 \pm 0.14$ & $13.48 \pm 0.03$ & $18.19 \pm 0.12$ & $15.43 \pm 0.04$ & $12.88 \pm 0.02$ & $18.99 \pm 0.18$ & $18.08 \pm 0.12$ \\
\hline F336W & $17.05 \pm 0.05$ & $11.77 \pm 0.02$ & $17.16 \pm 0.06$ & $13.20 \pm 0.02$ & $11.15 \pm 0.02$ & $17.29 \pm 0.06$ & $16.66 \pm 0.05$ \\
\hline F395N & $16.67 \pm 0.06$ & $12.15 \pm 0.02$ & $16.77 \pm 0.06$ & $13.50 \pm 0.03$ & $11.54 \pm 0.02$ & $16.90 \pm 0.07$ & $16.41 \pm 0.06$ \\
\hline F438W & $15.88 \pm 0.03$ & $11.41 \pm 0.02$ & $15.98 \pm 0.03$ & $12.58 \pm 0.02$ & $10.77 \pm 0.02$ & $15.72 \pm 0.03$ & $15.56 \pm 0.03$ \\
\hline $\mathrm{F} 467 \mathrm{M}$ & $\ldots$ & $11.07 \pm 0.02$ & $\ldots$ & $\ldots$ & $10.39 \pm 0.02$ & $\ldots$ & $\ldots$ \\
\hline F555W & $14.28 \pm 0.02$ & $\ldots$ & $14.35 \pm 0.02$ & $11.62 \pm 0.02$ & .. & $13.91 \pm 0.02$ & $14.05 \pm 0.02$ \\
\hline $\mathrm{F} 625 \mathrm{~W}$ & $13.25 \pm 0.02$ & $\ldots$ & $13.34 \pm 0.02$ & $10.87 \pm 0.02$ & $\ldots$ & $12.83 \pm 0.02$ & $13.06 \pm 0.02$ \\
\hline F631N & $\ldots$ & $9.96 \pm 0.02$ & $\ldots$ & $\ldots$ & $9.30 \pm 0.02$ & $\ldots$ & $\ldots$ \\
\hline F656N & $12.04 \pm 0.03$ & $9.39 \pm 0.02$ & $12.13 \pm 0.03$ & $10.30 \pm 0.02$ & $8.67 \pm 0.02$ & $11.71 \pm 0.03$ & $11.86 \pm 0.03$ \\
\hline F673N & $\ldots$ & $9.73 \pm 0.02$ & $\ldots$ & $\ldots$ & $9.09 \pm 0.02$ & $\ldots$ & $\ldots$ \\
\hline F775W & $11.69 \pm 0.02$ & $9.38 \pm 0.02$ & $11.95 \pm 0.02$ & $10.25 \pm 0.02$ & $8.82 \pm 0.02$ & $11.47 \pm 0.02$ & $11.73 \pm 0.02$ \\
\hline
\end{tabular}

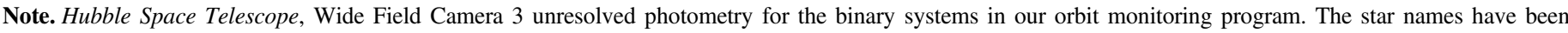

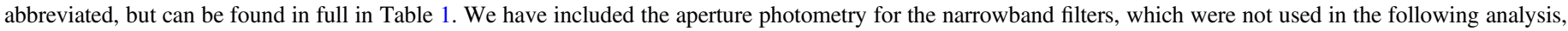
for completeness and future use.

Table 5

HST/WFC3 Resolved Photometry

\begin{tabular}{|c|c|c|c|}
\hline Property & RXJ $1550 \ldots$ & USco J1605... & GSC $6794-156$ \\
\hline MJD & 56031 & 56032 & 56032 \\
\hline$\rho$ (mas) & $65.8 \pm 0.2$ & $31.2 \pm 0.7$ & $71.8 \pm 0.4$ \\
\hline P.A. $\left({ }^{\circ}\right)$ & $90.1 \pm 1.2$ & $270.6 \pm 8.5$ & $115.1 \pm 1.8$ \\
\hline$\Delta \mathrm{F} 225 \mathrm{~W}$ & $\ldots$ & $\ldots$ & $1.71 \pm 0.35$ \\
\hline$\Delta \mathrm{F} 275 \mathrm{~W}$ & $\cdots$ & $\cdots$ & $1.29 \pm 0.31$ \\
\hline$\Delta \mathrm{F} 336 \mathrm{~W}$ & $1.41 \pm 0.33$ & $\cdots$ & $0.94 \pm 0.31$ \\
\hline$\Delta$ F390W & $1.68 \pm 0.25$ & $\cdots$ & $0.75 \pm 0.27$ \\
\hline$\Delta \mathrm{F} 395 \mathrm{~N}$ & $1.49 \pm 0.36$ & $\cdots$ & $0.81 \pm 0.48$ \\
\hline$\Delta \mathrm{F} 438 \mathrm{~W}$ & $1.76 \pm 0.38$ & $\cdots$ & $0.70 \pm 0.49$ \\
\hline$\Delta \mathrm{F} 467 \mathrm{M}$ & $\ldots$ & $\cdots$ & $0.46 \pm 0.23$ \\
\hline$\Delta \mathrm{F} 475 \mathrm{~W}$ & $2.31 \pm 0.17$ & $\cdots$ & $\cdots$ \\
\hline$\Delta \mathrm{F} 547 \mathrm{M}$ & $\ldots$ & $\cdots$ & $0.53 \pm 0.17$ \\
\hline$\Delta \mathrm{F} 555 \mathrm{~W}$ & $1.85 \pm 0.22$ & $0.52 \pm 0.40$ & $\ldots$ \\
\hline$\Delta \mathrm{F} 625 \mathrm{~W}$ & $1.60 \pm 0.15$ & $0.66 \pm 0.21$ & $\ldots$ \\
\hline$\Delta \mathrm{F} 631 \mathrm{~N}$ & $\ldots$ & $\ldots$ & $0.44 \pm 0.06$ \\
\hline$\Delta \mathrm{F} 656 \mathrm{~N}$ & $1.11 \pm 0.04$ & $0.71 \pm 0.14$ & $0.55 \pm 0.07$ \\
\hline$\Delta \mathrm{F} 673 \mathrm{~N}$ & $\ldots$ & $\ldots$ & $0.45 \pm 0.06$ \\
\hline$\Delta \mathrm{F} 775 \mathrm{~W}$ & $1.10 \pm 0.09$ & $0.68 \pm 0.18$ & $\ldots$ \\
\hline$\triangle \mathrm{F} 850 \mathrm{LP}$ & $0.95 \pm 0.02$ & $0.84 \pm 0.33$ & $0.52 \pm 0.06$ \\
\hline
\end{tabular}

Note. Hubble Space Telescope, Wide Field Camera 3 differential photometry for the binary systems in our orbit monitoring program. The star names have been abbreviated, but can be found in full in Table 1 . The first two rows list the separation and position angle of the companions at the epoch of HST observation. In general, we have not reported any magnitude difference with an uncertainty larger than $0.5 \mathrm{mag}$, or where the PSF fit was determined to have failed due to the close proximity of the two components. Magnitude differences were not derivable for the binary systems ScoPMS 17, GSC 6209-735, RX J1601.9-2008, and ROXs 47A, due to the high contrast or sub-pixel proximity of the components of these systems at the time of HST observations (12, 33.4, 16,39 mas, respectively).
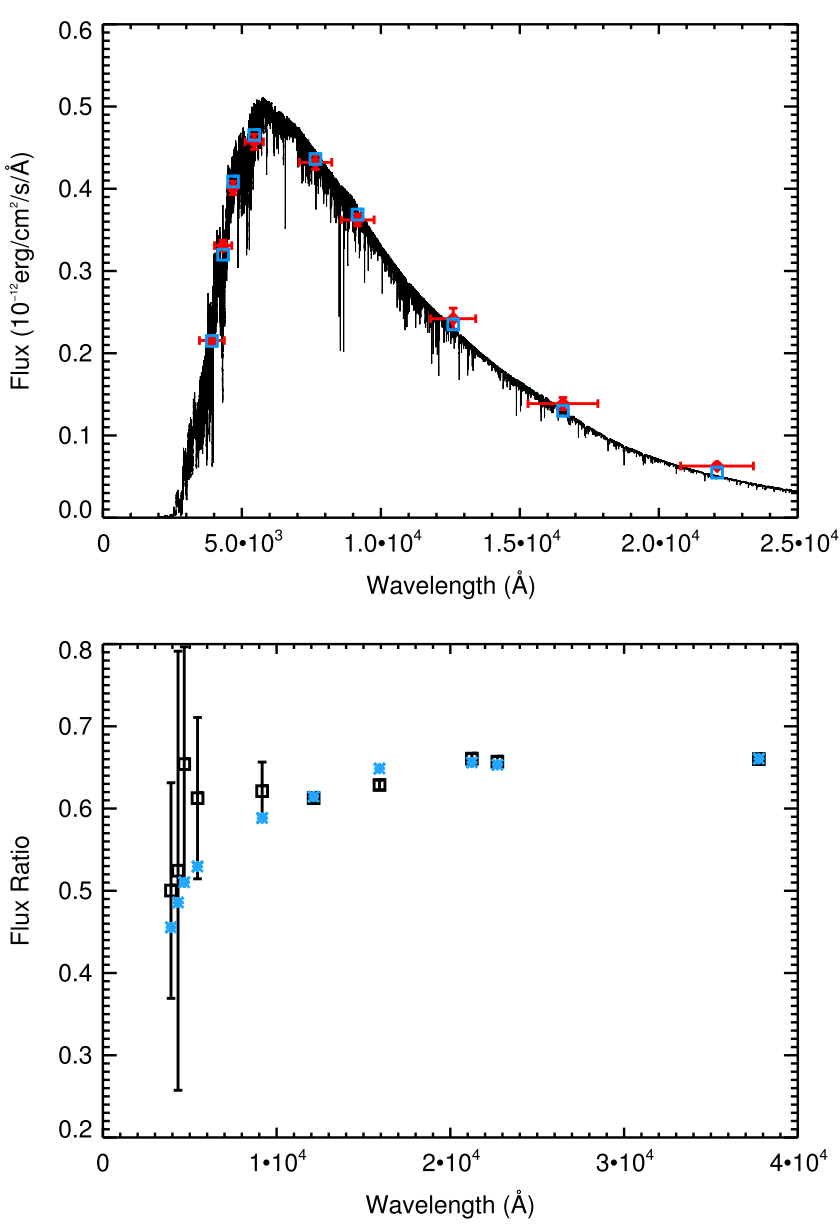

Figure 5. Binary SED fitted unresolved photometry (a) and flux ratios (b) for GSC 6794-156. The black line is the combined BT-Settl atmospheres at the two best-fit temperatures, the red points with error bars are the observed photometry, and the blue squares are the model photometry. The best-fit atmosphere shown here is a combination of two atmospheres with temperatures 5700 and $5350 \mathrm{~K}$. 

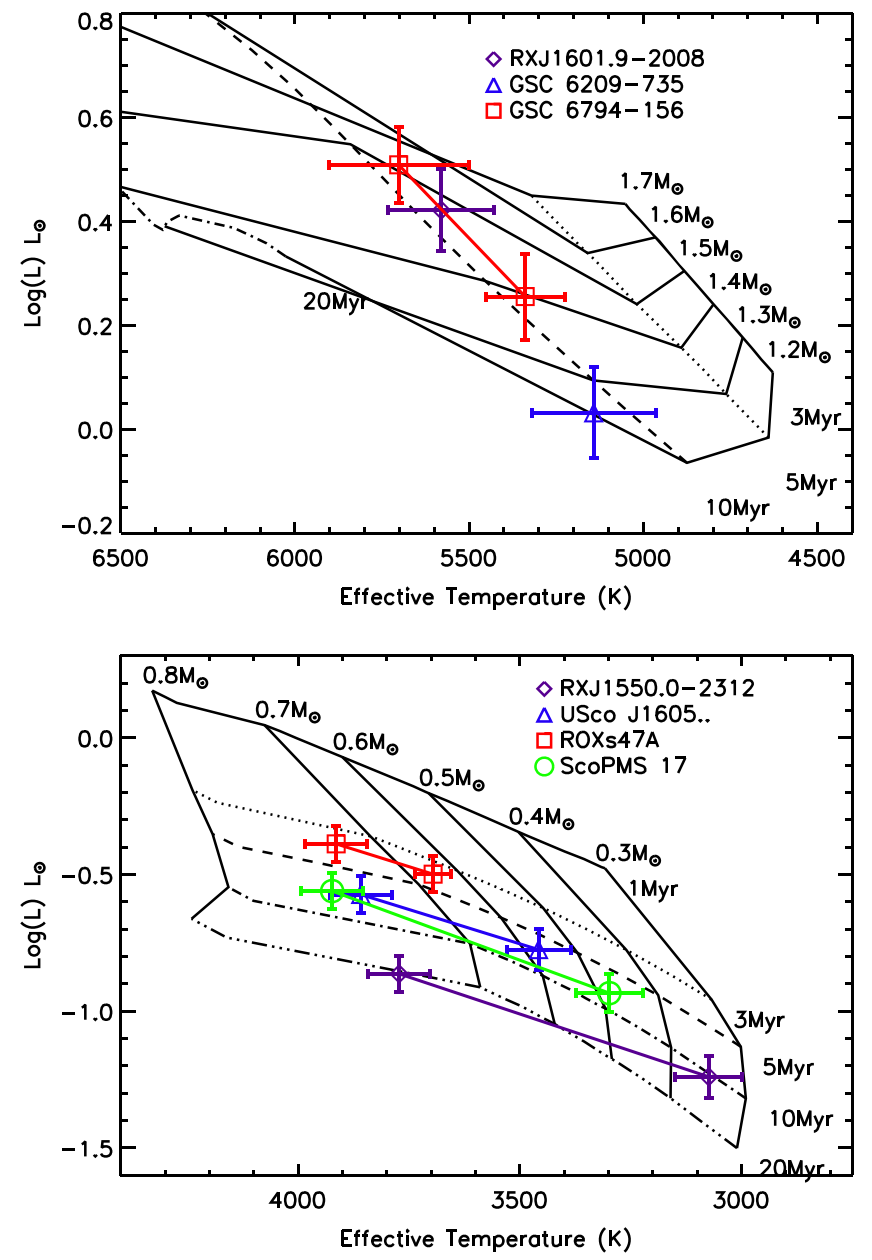

Figure 6. HR diagram positions for the binary system components in our orbit monitoring sample. Components of each binary system are displayed in a different color and with a different symbol, and are connected to the corresponding companions via a colored line. The black lines indicate the isochrone and isomass lines taken from the latest Padova models (Chen et al. 2014). For clarity, we display the higher-mass binaries in panel (a) and the M-type binary systems in panel (b).

given by the uncertainties in the data:

$$
\begin{aligned}
& P\left(\pi_{\star} \mid \pi_{m}\right) \propto \exp \left(-\frac{\left(\pi_{\star}-\pi_{m}\right)^{2}}{2 \sigma_{\pi_{\star}}}\right), \\
& P\left(M_{t} \pi^{3} \mid\left(M_{p+s}\right) \pi_{m}^{3}\right) \propto \exp \left(-\frac{\left(M_{t} \pi^{3}-\left(M_{p}+M_{s}\right) \pi_{m}^{3}\right)^{2}}{2 \sigma_{M_{t} \pi^{3}}^{2}}\right), \\
& P\left(M_{f(M)} \mid M_{s}^{3} M_{p+s}^{-2}\right) \propto \exp \left(-\frac{\left(M_{f(M)}-M_{s}^{3}\left(M_{p}+M_{s}\right)^{-2}\right)^{2}}{2 \sigma_{M_{f(M)}}^{2}}\right), \\
& P\left(m_{\star, i} \mid m_{s+p, i}\right) \propto \exp \left(-\frac{\left(m_{\star, i}-m_{s+p, i}\right)^{2}}{2 \sigma_{m_{\star, i}}^{2}}\right), \\
& P\left(\Delta m_{\star, j} \mid \Delta m_{j}\right) \propto \exp \left(-\frac{\left(\Delta m_{\star, j}-\Delta m_{j}\right)^{2}}{2 \sigma_{\Delta m_{\star, j}}^{2}}\right),
\end{aligned}
$$

where we have omitted the usual normalization factors for the purpose of readability. We then calculate the probability of a grid of model parameter sets using Equations (5) and (6) to determine the most likely value of age, component masses, and parallax. This was done with age ranging from 1 to $25 \mathrm{Myr}$ in steps of $1 \mathrm{Myr}$, and primary and secondary mass ranging from a minimum of $0.5 M_{\odot}$ to $2 M_{\odot}$ in steps of $0.01 M_{\odot}$. The model parallax was varied from 2 to 15 mas in steps of 0.1 mas, and the reddening parameter $E(B-V)$ was varied from 0 to 1 in steps of $0.05 \mathrm{mag}$. Once a likely solution was found, we decreased the step size and sampling range to fully sample the probability distribution, using steps of $0.25-0.5 \mathrm{Myr}$ in age and $0.005 M_{\odot}$ in mass, and interpolating between isochrones. We also added in quadrature an error of $0.05 \mathrm{mag}$ to all nonsimultaneous photometric measurements to account for the average variability of PMS dwarfs (Herbst et al. 2007).

To reduce the dependence of the results of our age estimation on the characteristics of any one particular set of model isochrones, or at least to illuminate the model dependence, we use the Padova (Girardi et al. 2002), the Dartmouth PMS model (Dotter et al. 2008), and the BT-Settl isochrones (Allard et al. 2011) to determine stellar properties of the binary systems in our Upper Scorpius sample. The Padova isochrones employ the PHOENIX BT-Settl model atmospheres (Allard \& Hauschildt 1995; Allard et al. 2011) for stars with effective temperatures cooler than $4000 \mathrm{~K}$ and ATLAS9 (Castelli \& Kurucz 2004) model atmosphere for the hotter stars. These atmospheres are used for both the $T_{\text {eff }}$ to synthetic color transformations and for establishing the relationship between temperature and the mean optical depth. This is then empirically adjusted based on colors of globular cluster stars (Chen et al. 2014). Similarly, the Dartmouth models use the PHOENIX model atmospheres directly without further correction for all stars cooler that $10,000 \mathrm{~K}$, which spans the temperature range of all the primary stars included in this study. The BT-Settl isochrones are constructed by interpolating the Allard et al. (2011) synthetic atmospheres over the Baraffe et al. $(1998,2003)$ model grids and are usable for stellar masses less than $1.4 M_{\odot}$.

There has been some issue with the color-magnitude relations for lower-mass stars (masses smaller than $\left.\sim 1.2 M_{\odot}\right)$. Models that closely reproduce observations in the near-IR and the redder optical colors often produce optical color significantly bluer than expected (An et al. 2008; Dotter et al. 2008). The empirical corrections applied in the Padova isochrones were introduced to mitigate this issue for mainsequence dwarfs, and so we expect the Padova isochrones to more closely reproduce the photometry of our young binary systems. It is important to note, however, that the effectiveness of these corrections for PMS stars is not clear.

The photometry available to us for fitting varies between objects, for the most part, $B, V, g, r$, and $i$ magnitudes from the APASS survey, which were of varying quality, and 2MASS near-IR magnitudes are available for all the target systems. The only absent photometry was the $g$ - and $r$-band APASS magnitudes for ScoPMS 17. As mentioned previously, ROXs 47A is a hierarchical triple system (Barsony et al. 2003), of which we are examining the inner dynamical system. The third component of the system, which is of comparable brightness to the primary, is still relatively close to the primary at $0 . " 79$, which means that the 2MASS and APASS photometry are contaminated and unusable. Tables 7 and 8 tabulate all publicly available photometry for the stars in our sample, and for 
Table 6

Model System Properties

\begin{tabular}{|c|c|c|c|c|c|c|c|c|}
\hline Name & $\begin{array}{l}T_{\text {eff,p }} \\
(\mathrm{K})\end{array}$ & $\begin{array}{c}T_{\text {eff,s }} \\
(\mathrm{K})\end{array}$ & $\begin{array}{c}L_{\mathrm{p}} \\
\left(L_{\odot}\right)\end{array}$ & $\begin{array}{c}L_{\mathrm{s}} \\
\left(L_{\odot}\right)\end{array}$ & $\begin{array}{l}\text { Age } \\
(\mathrm{Myr})\end{array}$ & $\begin{array}{c}M_{\mathrm{p}} \\
\left(M_{\odot}\right)\end{array}$ & $\begin{array}{c}M_{\mathrm{s}} \\
\left(M_{\odot}\right)\end{array}$ & $\begin{array}{c}D \\
(\mathrm{pc})\end{array}$ \\
\hline GSC 6794-156 & $5700 \pm 201$ & $5340 \pm 114$ & $3.544 \pm 0.594$ & $1.977 \pm 0.376$ & $9 \pm 3$ & $1.54 \pm 0.13$ & $1.42 \pm 0.11$ & $\overline{138 \pm 15}$ \\
\hline GSC 6209-735 & $5140 \pm 178$ & $\ldots$ & $1.283 \pm 0.258$ & $\ldots$ & $12 \pm 6$ & $1.23 \pm 0.14$ & $\ldots$ & $133 \pm 16$ \\
\hline USco J1605... & $3760 \pm 50$ & $3460 \pm 69$ & $0.213 \pm 0.033$ & $0.138 \pm 0.024$ & $6 \pm 1$ & $0.71 \pm 0.05$ & $0.57 \pm 0.05$ & $161 \pm 16$ \\
\hline ScoPMS 17 & $3860 \pm 36$ & $3300 \pm 58$ & $0.301 \pm 0.045$ & $0.132 \pm 0.022$ & $7 \pm 2$ & $0.72 \pm 0.05$ & $0.48 \pm 0.05$ & $136 \pm 15$ \\
\hline RX J1550.0-2312 & $3690 \pm 38$ & $3010 \pm 67$ & $0.316 \pm 0.048$ & $0.138 \pm 0.024$ & $16 \pm 3$ & $0.71 \pm 0.05$ & $0.35 \pm 0.05$ & $93 \pm 15$ \\
\hline
\end{tabular}

Note. System properties derived from fitting BT-Settl models to the binary system photometry and comparison. Due to the high contrast ratios for RX J1601.9-2008 and GSC 6209-735, only the primary temperature and luminosity were determined. The ages and masses are taken from comparison to the Padova isochrones.

Table 7

APASS Photometry

\begin{tabular}{|c|c|c|c|c|c|}
\hline Star & $B$ & V & $g$ & $r$ & $i$ \\
\hline RXJ $1550.0-2312$ & $15.613 \pm 0.479$ & $14.065 \pm 0.054$ & $14.760 \pm 0.337$ & $13.342 \pm 0.196$ & $12.059 \pm 0.102$ \\
\hline RXJ 1601.9-2008 & $11.333 \pm 0.043$ & $10.380 \pm 0.030$ & $10.985 \pm 0.187$ & $10.086 \pm 0.004$ & $9.637 \pm 0.011$ \\
\hline USco J160517.9-202420 & $15.858 \pm 0.052$ & $14.224 \pm 0.035$ & $15.059 \pm 0.042$ & $13.497 \pm 0.036$ & $12.400 \pm 0.140$ \\
\hline GSC $6209-735$ & $12.514 \pm 0.058$ & $11.403 \pm 0.049$ & $11.917 \pm 0.033$ & $11.012 \pm 0.053$ & $10.626 \pm 0.095$ \\
\hline GSC 6794-156 & $10.673 \pm 0.015$ & $9.775 \pm 0.085$ & $10.321 \pm 0.150$ & $9.499 \pm 0.201$ & $8.980 \pm 0.144$ \\
\hline ScoPMS 17 & $15.571 \pm 0.366$ & $13.833 \pm 0.095$ & $\cdots$ & $\cdots$ & $12.058 \pm 0.086$ \\
\hline ROXs 47A & $15.381 \pm 0.098$ & $13.611 \pm 0.095$ & $14.510 \pm 0.119$ & $12.835 \pm 0.092$ & $11.615 \pm 0.051$ \\
\hline
\end{tabular}

Note. The $B, V, g, r$, and $i$ magnitudes taken from APASS data release 9. Note that the photometry for ROXs 47A includes the wide tertiary component of the system, and so are not used in our fitting procedure below.

completeness, we include ROXs 47A in the table, though we do not use this photometry in the fitting procedure described above. As mentioned above, we also incorporated our HST WFC3 wide-band photometry and differential photometry obtained in 2012. For all the targets, we excluded the filters F225W, F275W, and F336W because this wavelength range is highly sensitive to the activity of the PMS stars in question, and so cannot be reliably used to fit to models. We also empirically tested the effects of the F390W and F438W photometry on the fits for the M-type stars in the sample but found that the inclusion or removal of these data did not significantly change the output results.

\subsection{Estimated Stellar Properties}

Computation of the posterior probability for our Bayesian models yields a five-dimensional space of probabilities, one dimension for each model parameter, which can be reduced to lower dimensionality by marginalizing over uncorrelated model parameters. We found none of our model parameters were strongly correlated, with the reddening parameter showing some correlation with the other parameters for some of the stars in our sample. This is expected given the possibility of degeneracy between distance, system mass, and extinction as a function of age. We produce one-dimensional probability densities for the parameters of our model, and then determine the intervals that contain the most likely values of each parameter. For all of the binary systems we have studied, a single most probable set of stellar parameters can be determined, with a corresponding uncertainty range that varied in size between systems. Figure 7 displays the probability of each model parameter for the star USco J160517.9-202420.

Given these output distributions, we then calculate $1 \sigma$ Bayesian "credible" intervals for each parameter, which can be
Table 8

2MASS Photometry

\begin{tabular}{lrcc}
\hline \hline Star & \multicolumn{1}{c}{$J$} & \multicolumn{1}{c}{$H$} & $K$ \\
\hline RXJ 1550.0-2312 & $9.885 \pm 0.024$ & $9.215 \pm 0.023$ & $8.930 \pm 0.023$ \\
RXJ 1601.9-2008 & $8.350 \pm 0.020$ & $7.808 \pm 0.026$ & $7.672 \pm 0.020$ \\
USco J160517.9- & $10.154 \pm 0.022$ & $9.349 \pm 0.024$ & $9.143 \pm 0.019$ \\
$\quad$ 202420 & & & \\
GSC 6209-735 & $9.158 \pm 0.030$ & $8.603 \pm 0.042$ & $8.426 \pm 0.020$ \\
GSC 6794-156 & $7.779 \pm 0.027$ & $7.280 \pm 0.027$ & $7.084 \pm 0.018$ \\
ScoPMS 17 & $9.932 \pm 0.024$ & $9.235 \pm 0.026$ & $8.992 \pm 0.02$ \\
ROXs 47A & $9.245 \pm 0.024$ & $8.351 \pm 0.031$ & $7.929 \pm 0.061$ \\
\hline
\end{tabular}

Note. The infrared are taken from the 2MASS catalog. Note that the photometry for ROXs 47A includes the wide tertiary component of the system, and so are not used in our fitting procedure below.

found in Table 9. Note that the BT-Settl isochrones were only applied to the binary systems in our sample where the component masses were expected to be less than $1 M_{\odot}$. We find that the most likely system parameters output from the Bayesian method agree with the estimates obtained from the conventional SED fitting and HR diagram positions. Below, we individually summarize the results for each binary system, detailing the clarity of each fit.

\subsubsection{GSC $6794-156$}

Both the Padova and Dartmouth model fits produce a system age of $\sim 10.5 \mathrm{Myr}$ for GSC 6794-156, which is consistent with the recent Pecaut et al. (2012) age estimation for Upper Scorpius. In the other four parameters, the models agree closely. The best-fit system parallax for both models is $7.3 \pm 0.2 \mathrm{mas}$, which is consistent with the mean distance and distance spread in Upper Scorpius. We find that the Padova 

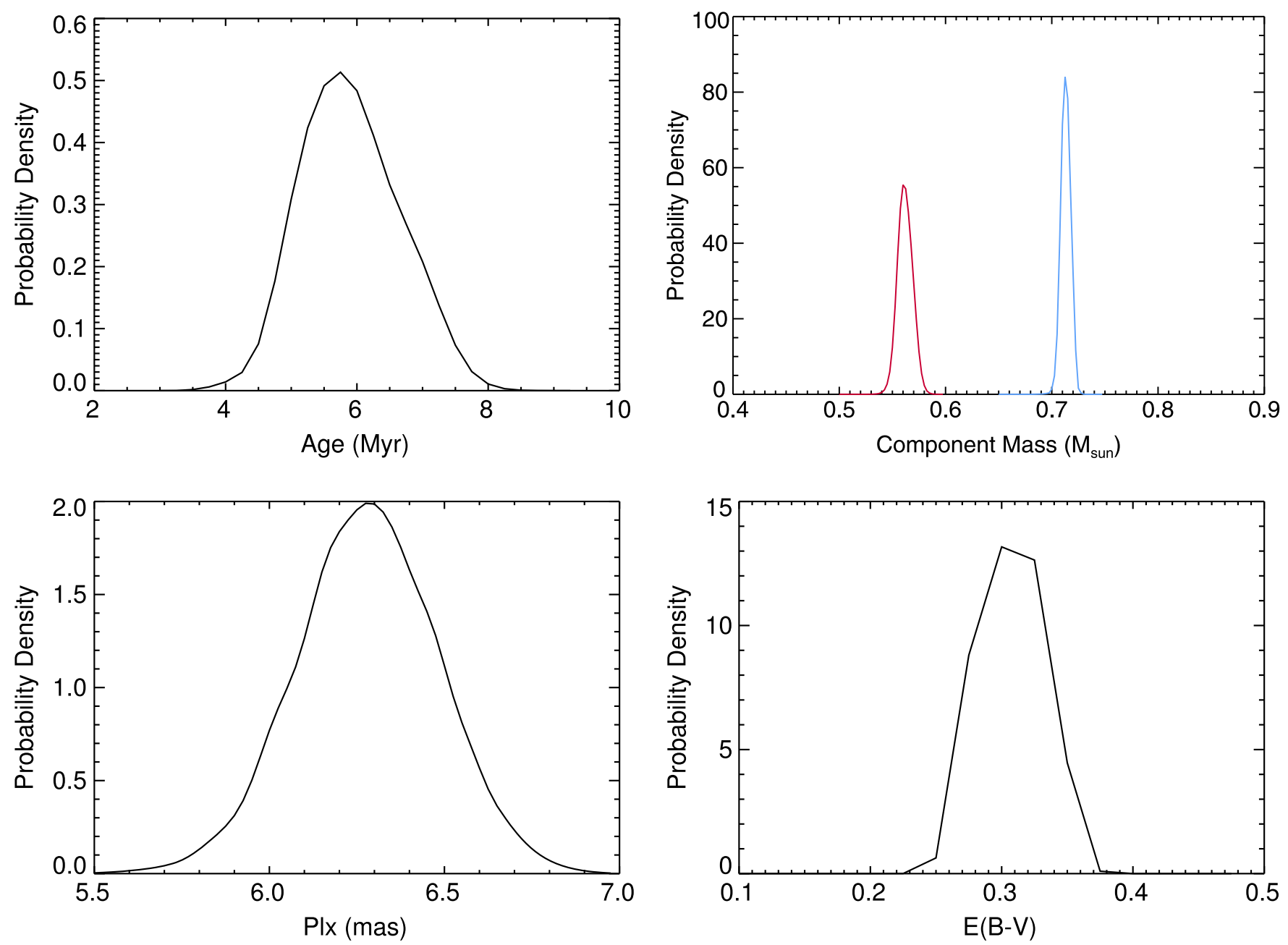

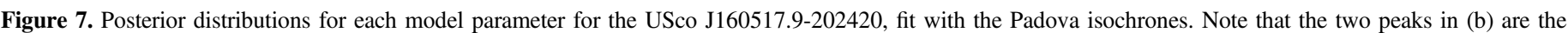

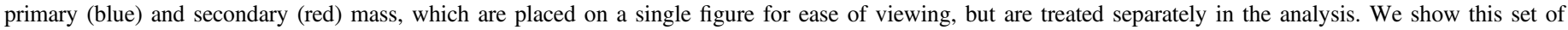

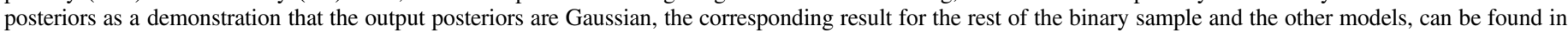
Table 9.

and Dartmouth models produce a most likely reddening parameter of $\sim E(B-V)=0.43 \mathrm{mag}$. Given both the $\Delta J$ and $\Delta K$ values from the Keck NIRC2 aperture masking (see Table 2), we can estimate the expected extinction for this system using standard tables of template photometry for young systems, with some uncertainty produced by the unclear spectral type of the primary. The tables of Bessell \& Brett (1988) give an intrinsic $J-K$ color of of $0.43 \mathrm{mag}$ for the approximately G6 primary of system this system. The observed color, corrected for the presence of the companion using the aperture masking contrasts, is $0.617 \mathrm{mag}$, which then yields a value of $E(B-V) \sim 0.4$ for the system. Similar estimation using the intrinsic colors for young stars from Pecaut \& Mamajek (2013) produces a value of $E(B-V) \sim 0.2-0.4$ mag. Both of these values are consistent with our determination for the system.

\subsubsection{RXJ 1601.9-2008}

Both the Padova and Dartmouth models produce consistent ages of 12 Myr for the RXJ 1601.9-2008 binary system, and we note that as with the other G-type binary system in our sample (GSC 6794-156) the Padova age estimate is slightly older than that of the Dartmouth model. The other four system parameters agree closely between the models, and we note that both RXJ 1601.9-2008 and GSC 6794-156 have very similar primary component masses. This is expected given the similar spectral types for these two systems (G5 and G6, respectively).

\subsubsection{GSC 6209-735}

The visual orbit for GSC 6209-735 is the least wellconstrained in our sample due to the large contrast ratio between the primary and secondary $(\Delta H=3.05 \mathrm{mag})$ and the small angular separation. Fortunately, GSC 6209-735 has been known to be a single-lined spectroscopic binary for some time, and the single-component radial velocity orbit has been previously determined (Guenther et al. 2007). We included the single-lined orbit information in the model fit as a second mass observable as described above and were able to produce posteriors for the stellar parameters that are relatively wellconstrained. We find that the component masses and the system parallax determined for the three models agree within the uncertainties, with the system parallax of $7.9 \pm 0.2$ mas being consistent with Upper Scorpius membership. The Dartmouth and BT-Settl models produce the most probable ages of 23-26 Myr, while the Padova isochrones produce an age of $\sim 19$ Myr. The possible age solutions for this binary system are 
Table 9

Bayesian Estimated Stellar Parameters

\begin{tabular}{|c|c|c|c|c|c|c|c|}
\hline Name & Model & $\begin{array}{l}\text { Age } \\
(\mathrm{Myr})\end{array}$ & $\begin{array}{c}M_{\mathrm{p}} \\
\left(M_{\odot}\right)\end{array}$ & $\begin{array}{c}M_{\mathrm{s}} \\
\left(M_{\odot}\right)\end{array}$ & $\begin{array}{c}\pi \\
\text { (mas) }\end{array}$ & $\begin{array}{c}E(B-V) \\
(\mathrm{mag})\end{array}$ & $\chi_{\mathrm{r}}^{2}$ \\
\hline \multirow[t]{2}{*}{ GSC 6794-156 } & $P$ & $10.5_{-1.7}^{+1.3}$ & $1.51 \pm 0.1$ & $1.41 \pm 0.1$ & $7.3 \pm 0.2$ & $0.40 \pm 0.12$ & 6.4 \\
\hline & $\mathrm{D}$ & $10.2_{-1.0}^{+1.6}$ & $1.46 \pm 0.2$ & $1.38 \pm 0.2$ & $7.3 \pm 0.2$ & $0.37 \pm 0.10$ & 7.3 \\
\hline \multirow{2}{*}{ RXJ 1601.9-2008 } & $\mathrm{P}$ & $12.8 \pm 1.5$ & $1.46 \pm 0.05$ & $0.82 \pm 0.04$ & $6.8 \pm 0.2$ & $0.53 \pm 0.10$ & 6.8 \\
\hline & D & $11.5 \pm 2.2$ & $1.45 \pm 0.02$ & $0.77 \pm 0.04$ & $6.8 \pm 0.3$ & $0.43 \pm 0.07$ & 7.3 \\
\hline \multirow[t]{2}{*}{ GSC $6209-735$} & $\mathrm{P}$ & $18.8 \pm 5.6$ & $1.14 \pm 0.09$ & $0.45 \pm 0.02$ & $8.0 \pm 0.7$ & $0.41 \pm 0.09$ & 5.6 \\
\hline & D & $23.8_{-6.8}^{+5.8}$ & $1.23 \pm 0.15$ & $0.27 \pm 0.03$ & $8.1 \pm 1.1$ & $0.57 \pm 0.09$ & 8.6 \\
\hline \multirow{2}{*}{ USco J1605... } & $\mathrm{D}$ & $5.4 \pm 0.8$ & $0.61 \pm 0.03$ & $0.45 \pm 0.02$ & $6.6 \pm 0.3$ & $0.29 \pm 0.04$ & 1.7 \\
\hline & B & $6.5 \pm 0.7$ & $0.65 \pm 0.03$ & $0.49 \pm 0.03$ & $6.5 \pm 0.3$ & $0.29 \pm 0.02$ & 1.3 \\
\hline \multirow[t]{3}{*}{ ScoPMS 17} & $\mathrm{P}$ & $7.1 \pm 0.9$ & $0.71 \pm 0.01$ & $0.45 \pm 0.01$ & $7.5 \pm 0.1$ & $0.21 \pm 0.03$ & 2.3 \\
\hline & D & $6.5 \pm 0.7$ & $0.59 \pm 0.03$ & $0.35 \pm 0.02$ & $8.0 \pm 0.2$ & $0.21 \pm 0.02$ & 1.6 \\
\hline & B & $7.4 \pm 0.7$ & $0.59 \pm 0.03$ & $0.36 \pm 0.02$ & $8.0 \pm 0.2$ & $0.19 \pm 0.03$ & 1.4 \\
\hline & B & $5.2 \pm 1.6$ & $0.85 \pm 0.11$ & $0.78 \pm 0.08$ & $6.8 \pm 0.3$ & $0.48 \pm 0.08$ & 7.1 \\
\hline
\end{tabular}

Note. The models Padova (P), Dartmouth (D), and BT-Settl (B) refer to the Girardi et al. (2002), Dotter et al. (2008), and Allard et al. (2011) model grids, respectively. The final column lists the model best-fit reduced $\chi^{2}$ value.

significantly older than the other PMS binary systems in our sample and places GSC 6209-735 as a potential member of the older Upper Centaurus Lupus subgroup of Sco-Cen. The estimated parallax and location of GSC 6209-735 near the center of Upper Scorpius are consistent with both Upper Scorpius and UCL. We also note that GSC 6209-735 is the only K-type star in our sample and shows the largest age discrepancy of the binary systems we have monitored.

\subsubsection{USco J160517.9-202420}

We find that the best-fit model parameters for USco J160517.9-202420 indicate that it is a young binary system of age $\sim 6 \mathrm{Myr}$, with the three model fits producing age and parallax estimates that agree very closely. There is some difference in the best-fit mass between the models. The Padova model produces primary and secondary masses that are significantly larger than the corresponding Dartmouth and BT-Settl model fits. The study in which this star was identified as a Sco-Cen member estimates $E(B-V)=0.3$ (Preibisch et al. 2002), and estimation using spectral type (M3) and $J-K$ color yields $E(B-V) \sim 0.3$ using the intrinsic color tables of Bessell \& Brett (1988). Both these estimations are consistent with our estimates.

\subsubsection{ScoPMS 17}

All three models produce an age consistent with $\sim 7$ Myr for ScoPMS 17; however, as with USco J160517.9-202420, the Padova models produce significantly larger masses for both the primary and secondary components of the binary system. Estimation from the color tables using the spectral type (M1) and the $J-K$ color gives a value of $E(B-V) \sim 0.2$ (Bessell $\&$ Brett 1988), which is consistent with out model fits.

\subsection{6. $R X J 1550.0-2312$}

From the original orbital solution for RXJ 1550.0-2312, there was clear evidence that the system was significantly closer than the median Upper Scorpius parallax of $\sim 6.9$ mas (see Table 3). Upon applying the Bayesian fitting method described above, we found a peak in the system parallax PDF beyond 10 mas, and so we removed the input prior system parallax of $7.5 \pm 1.6$ mas and refit the data for the three models. The model age fits for RXJ 1550.0-2312 are significantly older than those of the other M-type Upper Scorpius binary systems we have studied ( $>14 \mathrm{Myr}$ ), and we note that the Padova age estimate is older still than that of the other models. As with the other M-type systems in this study, the Padova mass estimates are significantly larger than that of the Dartmouth or BT-Settl models. RXJ 1550.0-2312 sits in the region of sky $(l, b=347.8,23.7)$ traditionally considered the border between Upper Scorpius and the older ( 16 Myr) UCL subgroup of Sco-Cen (de Zeeuw et al. 1999). Given the foreground distance and older age estimation found here, we suggest that RXJ $1550.0-2312$ is a member of the UCL subgroup.

\subsubsection{ROXs $47 A$}

We expected ROXs 47A to be the most difficult M-type system for the models to accurately reproduce, given the very young age and disk presence. The Bayesian fitting procedure produced a very young and highly reddened fit for ROXs 47A, with an estimated age $<4$ Myr for the Padova and Dartmouth models. The estimated parallaxes for both the Padova and Dartmouth models (7.8 mas and 7.7 mas, respectively) are consistent with ROXs 47A being a member of the very young $\rho$-Ophiuchus star-forming region, which is located at $(\alpha$, $\delta)=\left(16^{\mathrm{h}} 28^{\mathrm{m}},-24^{\circ} 33^{\prime \prime}\right)$ and a distance of $\sim 130 \mathrm{pc}$, i.e., slightly closer than the Upper Scorpius subgroup. The BT-Settl models produce significantly different fits to the data, with very 
large component masses of $0.85 M_{\odot}$ and $0.78 M_{\odot}$, respectively, and a parallax of $6.9 \pm 0.2$ mas. This parallax is inconsistent with the distance to Ophiuchus, and the estimated age of $5.2 \pm 0.5 \mathrm{Myr}$ is significantly older than the mean age of 2.1 Myr of the Ophiuchus PMS stars (McClure et al. 2010). All three fits produce extinction values consistent with extinction value of $E(B-V)=0.52 \mathrm{mag}$ estimated by McClure et al. (2010).

\section{EVALUATION OF THE PMS MODELS}

With the results of the Bayesian model fitting procedure described above, it is possible to make informed statements about the behavior of the models for predicting stellar properties of young stars of different mass and spectral type and the difference in outcome between the varieties of models for evaluating a single binary system.

First, we see that for the M-type binary systems, the fitted component masses are highly dependent on the choice of model, with the Padova models producing significantly larger component masses than the Dartmouth or BT-Settl models, while the other four parameters are in general agreement. This is consistent with recent observations of UScoCTIO 5, an M4.5 eclipsing binary in Upper Scorpius. Kraus et al. (2015) found the model masses produced for UScoCTIO 5 were overestimated by the Padova models and underestimated by the Dartmouth and Baraffe models (Baraffe et al. 2015). In contrast to this trend, the fitted parameters for the G-type stars in our sample agree very closely between the Padova and Dartmouth models, which predict very similar component masses and system parallaxes.

A number of the binary systems we have characterized appear to be younger than $10 \mathrm{Myr}$ according to the models, while other binary systems in our sample are of an age $>10 \mathrm{Myr}$. The Pecaut et al. (2012) study, which used photometry for B-, A-, F-, and G-type stars, estimated the age of the Upper Scorpius subgroup to be $11 \pm 2 \mathrm{Myr}$, which is significantly different than the age determinations for the younger stars in our work and the age estimations in previous work (de Geus 1992; Preibisch et al. 2002).

Most importantly, we find that across the three models, there is a significant dichotomy in the estimated ages, with the G-type members appearing older ( $12 \mathrm{Myr})$ compared to the M-type members ( $\sim 7 \mathrm{Myr})$. This trend was broadly mirrored in the conventional HR diagram position ages from the binary SED fits. In this comparison, we exclude RX J1550.0-2312 because it has distance and age estimates consistent with the older Upper Centaurus Lupus subgroup of Sco-Cen and GSC 6209-735, which appears discrepantly too old to be a member of Upper Scorpius and is the only K-type star in our study, making attribution of the age discrepancy to either the models or membership difficult. We have also removed ROXs 47A from this comparison due to it's expected youth as a member of Ophiuchus star-forming region. The apparent age difference between the remaining four G- and M-type binary systems we see here is consistent with previous HR diagram ages for different spectral type populations in Upper Scorpius. Preibisch et al. (2002) found that the M-type members in Upper Scorpius had a mean age between 3 and 5 Myr based on HR diagram estimation, and more recent work involving a new sample of Upper Scorpius PMS M-type members and modern isochrones found similar results, with the later M-type members showing

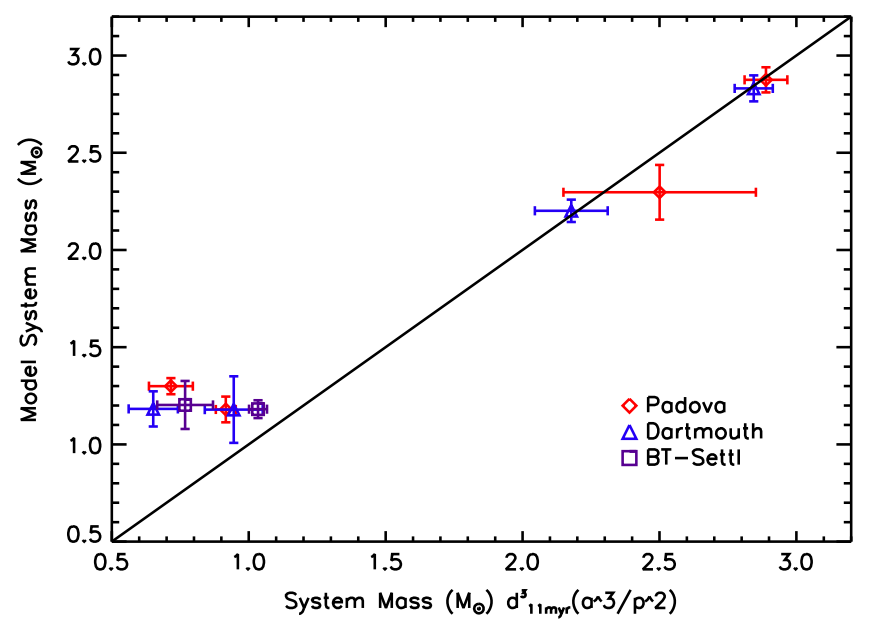

Figure 8. Measured system mass and model system mass at a fixed age of $11 \mathrm{Myr}$ for the two G-type and two M-type stars in our sample that are clear USco members. The measured system mass in the $x$-axis is derived from the orbital semimajor axis and period but is dependent of the model distance at the fixed age of $11 \mathrm{Myr}\left(d_{11 M y r}^{3} a^{3} / p^{2}\right)$. The model system masses for the M-type stars are significantly overestimated compared to the measured masses, while the G-type stars show very close agreement between the model fits and the data.

an overall younger age than K-type members (Rizzuto et al. 2015).

The most recent main-sequence A-type, PMS F-type, and PMS G-type member age estimates are $9 \pm 2 \mathrm{Myr}$, $13 \pm 1 \mathrm{Myr}$, and $10 \pm 3 \mathrm{Myr}$, respectively (Pecaut et al. 2012), all of which broadly agree with our Bayesian age estimate for the G-type binaries in our orbit monitoring sample. We thus conclude that the evolutionary models for the M-type stars, which we find to produce ages of $\sim 7 \mathrm{Myr}$, do not adequately reproduce the descent toward the main sequence along the Hayashi track (Hayashi 1961). In particular, this is equivalent to an underprediction of the luminosity of a PMS M-type star of a given mass at a given PMS age, with the typical luminosity underprediction of $0.08-0.15$ dex depending on the particular model and the stellar mass.

Given that distance is a free parameter in our analysis, it is difficult to disentangle whether the age discrepancy is caused by a miscalibration in the temperature scales of the models or the luminosity evolution of the star in time. In light of the recent study of the Upper Scorpius M-type eclipsing binary UScoCTIO 5 (Kraus et al. 2015), which indicates that in the case of a luminosity-independent test, the model luminosities are generally consistent with measurements, while the temperature predictions are incorrect, we recast the discrepancies in age that we observe in terms of effective temperature. Assuming that varying the system distance can produce agreement in both luminosity and system masses for a given system, this luminosity discrepancy is equivalent to a $100-300 \mathrm{~K}$ overestimation of the effective temperature of the binary components depending on stellar mass. This is clear in Figure 6, where shifting the isochrone grid to cooler temperatures gives older ages for the M-type binary system components.

To visualize the discrepancy between the models and the data in terms of the component masses, we fix the system age in our Bayesian fitting procedure to $11 \mathrm{Myr}$ and allow the component masses, parallax, and reddening parameter to be varied. We include in this comparison the two G-type stars 
GSC 6794-156 and RXJ 1601.9-2008 and the two clear M-type members of USco, J160517.9-202420 and ScoPMS 17. Figure 8 displays the fitted model system mass at $11 \mathrm{Myr}$ compared to the measured system mass at the best-fit $11 \mathrm{Myr}$ distance for each system. For the G-type stars, we find, as expected, that the model system masses agree closely with the measured system masses. The model system masses for the M-type binary systems are systematically larger, on the order of $0.2-0.4 M_{\odot}$, than the measured values.

The primary limitation in pinpointing the exact miscalibration in the sub-solar regime of the pre-main-sequence models is the absence of a precise measure of the binary system distances. The best available measure at this time is association membership ( $\pm 15 \mathrm{pc})$ and does not allow disentanglement of the degeneracies between luminosity, dynamical mass, and distance. The upcoming availability of high-precision parallaxes from the GAIA mission, combined with the orbital precision obtained for these systems, will break the current degeneracies in what could be causing the M-dwarf discrepancies.

\section{SUMMARY}

We have presented astrometric orbits and HST WFC3 photometry of seven G-, K-, and M-type binary systems in the young ( 10 Myr) Upper Scorpius subgroup of Sco-Cen. Using the orbital parameters and multi-band photometry, we have determined estimated system parameters based on various model isochrones using a Bayesian fitting technique. The model stellar properties for the seven binary systems allow us to conclude that:

1. The model isochronal ages derived from fitting to the Padova, Dartmouth, and BT-Settl isochrones for the G-type binary systems is $\sim 11.5 \mathrm{Myr}$, which is closely consistent with the latest HR diagram age for Upper Scorpius (Pecaut et al. 2012).

2. The mass predictions for the M-type binary systems differ between the models, with the Padova models predicting significantly larger binary component masses then the Dartmouth and BT-Settl isochrones.

3. For the M-type binary systems, the isochronal ages are $\sim 7 \mathrm{Myr}$, which is significantly younger than expected (11 Myr) and indicates calibration issues in the models for the M-type regime. This age discrepancy is equivalent to a luminosity underprediction of $0.08-0.15 \mathrm{dex}$, or an effective temperature overprediction of $100-300 \mathrm{~K}$. This suggests both the possibility of an uncertain temperature scale or further calibration issues in the mass-radius relation.

Some of the work presented here is based on observations made with the NASA/ESA Hubble Space Telescope, obtained from the data archive at the Space Telescope Science Institute. STScI is operated by the Association of Universities for Research in Astronomy, Inc., under NASA contract NAS 526555. Some of the data presented herein were obtained at the W.M. Keck Observatory, which is operated as a scientific partnership among the California Institute of Technology, the University of California, and the National Aeronautics and Space Administration. The Observatory was made possible by the generous financial support of the W.M. Keck Foundation. The authors wish to recognize and acknowledge the very significant cultural role and reverence that the summit of Mauna Kea has always had within the indigenous Hawaiian community. We are most fortunate to have the opportunity to conduct observations from this mountain.

\section{REFERENCES}

Allard, F., \& Hauschildt, P. H. 1995, ApJ, 445, 433

Allard, F., Homeier, D., \& Freytag, B. 2011, in ASP Conf. Ser. 448, 16th Cambridge Workshop on Cool Stars, Stellar Systems, and the Sun, ed. C. Johns-Krull, M. K. Browning, \& A. A. West (San Francisco, CA: ASP), 91

An, D., Johnson, J. A., Clem, J. L., et al. 2008, ApJS, 179, 326

Ardila, D., Martín, E., \& Basri, G. 2000, AJ, 120, 479

Baraffe, I., Chabrier, G., Allard, F., \& Hauschildt, P. H. 1998, A\&A, 337, 403

Baraffe, I., Chabrier, G., Barman, T. S., Allard, F., \& Hauschildt, P. H. 2003, A\&A, 402, 701

Baraffe, I., Homeier, D., Allard, F., \& Chabrier, G. 2015, A\&A, 577, A42

Barsony, M., Koresko, C., \& Matthews, K. 2003, ApJ, 591, 1064

Bessell, M. S., \& Brett, J. M. 1988, PASP, 100, 1134

Carpenter, J. M., Mamajek, E. E., Hillenbrand, L. A., \& Meyer, M. R. 2009, ApJ, 705, 1646

Castelli, F., \& Kurucz, R. L. 2004, arXiv:astro-ph/0405087

Chen, C. H., Mamajek, E. E., Bitner, M. A., et al. 2011, ApJ, 738, 122

Chen, Y., Girardi, L., Bressan, A., et al. 2014, MNRAS, 444, 2525

de Geus, E. J. 1992, A\&A, 262, 258

de Zeeuw, P. T., Hoogerwerf, R., de Bruijne, J. H. J., Brown, A. G. A., \& Blaauw, A. 1999, AJ, 117, 354

Dotter, A., Chaboyer, B., Jevremović, D., et al. 2008, ApJS, 178, 89

Ekström, S., Georgy, C., Eggenberger, P., et al. 2012, A\&A, 537, A146

Garcia, E. V., Dupuy, T. J., Allers, K. N., Liu, M. C., \& Deacon, N. R. 2015, ApJ, 804, 65

Girardi, L., Bertelli, G., Bressan, A., et al. 2002, A\&A, 391, 195

Guenther, E. W., Esposito, M., Mundt, R., et al. 2007, A\&A, 467, 1147

Hartig, G. F. 2008, WFC3 UVIS Shutter Vibration-Induced Image Blur, Tech. Rep., 2008-44

Hayashi, C. 1961, PASJ, 13, 450

Herbst, W., Eislöffel, J., Mundt, R., \& Scholz, A. 2007, in Protostars and Planets V, ed. B. Reipurth, D. Jewitt, \& K. Keil (Tucson, AZ: Univ. Arizona Press), 951

Hillenbrand, L. A., \& White, R. J. 2004, ApJ, 604, 741

Ireland, M. J., Kraus, A., Martinache, F., Law, N., \& Hillenbrand, L. A. 2011, ApJ, 726, 113

Köhler, R., Kunkel, M., Leinert, C., \& Zinnecker, H. 2000, A\&A, 356, 541

Kouwenhoven, M. B. N., Brown, A. G. A., Portegies Zwart, S. F., \& Kaper, L. 2007, A\&A, 474, 77

Kraus, A. L., Cody, A. M., Covey, K. R., et al. 2015, arXiv:1505.02446

Kraus, A. L., \& Hillenbrand, L. A. 2007, ApJ, 662, 413

Kraus, A. L., Ireland, M. J., Martinache, F., \& Hillenbrand, L. A. 2011, ApJ, 731,8

Kraus, A. L., Ireland, M. J., Martinache, F., \& Lloyd, J. P. 2008, ApJ, 679, 762

Krist, J. E., Hook, R. N., \& Stoehr, F. 2011, Proc. SPIE, 8127, 81270J

Lada, C. J., \& Lada, E. A. 2003, ARA\&A, 41, 57

Lafrenière, D., Jayawardhana, R., \& van Kerkwijk, M. H. 2008, ApJL, 689, L153

Lafrenière, D., Marois, C., Doyon, R., \& Barman, T. 2009, ApJL, 694, L148

Luhman, K. L., \& Mamajek, E. E. 2012, ApJ, 758, 31

Luhman, K. L., Stauffer, J. R., Muench, A. A., et al. 2003, ApJ, 593, 1093

Mamajek, E. E., Meyer, M. R., \& Liebert, J. 2002, AJ, 124, 1670

Martín, E. L., Delfosse, X., \& Guieu, S. 2004, AJ, 127, 449

McClure, M. K., Furlan, R., Manoj, P., et al. 2010, ApJS, 188, 75

Pecaut, M. J., \& Mamajek, E. E. 2013, ApJS, 208, 9

Pecaut, M. J., Mamajek, E. E., \& Bubar, E. J. 2012, ApJ, 746, 154

Preibisch, T., Brown, A. G. A., Bridges, T., Guenther, E., \& Zinnecker, H. 2002, AJ, 124, 404

Preibisch, T., Guenther, E., \& Zinnecker, H. 2001, AJ, 121, 1040

Preibisch, T., Guenther, E., Zinnecker, H., et al. 1998, A\&A, 333, 619

Preibisch, T., \& Mamajek, E. 2008, in Handbook of Star Forming Regions, Volume II: The Southern Sky, ed. B. Reipurth (ASP Monograph Publications, Vol. 5.; San Francisco, CA: ASP), 235

Rajan, A. E. 2010, WFC3 Data Handbook v. 2.1

Rizzuto, A. C., Ireland, M. J., \& Kraus, A. L. 2015, MNRAS, 448, 2737

Rizzuto, A. C., Ireland, M. J., \& Robertson, J. G. 2011, MNRAS, 416, 3108 Rizzuto, A. C., Ireland, M. J., \& Zucker, D. B. 2012, MNRAS, 421, L97

Rizzuto, A. C., Ireland, M. J., Robertson, J. G., et al. 2013, MNRAS, 436, 1694 
Savage, B. D., \& Mathis, J. S. 1979, ARA\&A, 17, 73

Schaefer, G. H., Prato, L., Simon, M., \& Patience, J. 2014, AJ, 147, 157

Simon, M., Schaefer, G. H., Prato, L., et al. 2013, ApJ, 773, 28

Simón-Díaz, S., Herrero, A., Esteban, C., \& Najarro, F. 2006, A\&A, 448, 351

Slesnick, C. L., Carpenter, J. M., \& Hillenbrand, L. A. 2006, AJ, 131, 3016

Song, I., Zuckerman, B., \& Bessell, M. S. 2012, AJ, 144, 8

Stassun, K. G., Feiden, G. A., \& Torres, G. 2014, NewAR, 60, 1
Strassmeier, K. G. 2009, A\&ARv, 17, 251

Tango, W. J., Davis, J., Ireland, M. J., et al. 2006, MNRAS, 370, 884

Walter, F. M., Vrba, F. J., Mathieu, R. D., Brown, A., \& Myers, P. C. 1994, AJ, 107, 692

Wright, E. L., Eisenhardt, P. R. M., Mainzer, A. K., et al. 2010, AJ, 140,1868

Yelda, S., Lu, J. R., Ghez, A. M., et al. 2010, ApJ, 725, 331 